\title{
Asymmetric Hydrogenation of tri-substituted Olefins with Ir-Phosphine Thiazole Complexes: A further Investigation of the Ligand Structure
}

\section{Supporting information}

All reactions were conducted under argon using dried glassware and magnetic stirring. THF was freshly distilled from sodium-benzophenone ketyl under $\mathrm{N}_{2}$ prior to use. $\mathrm{CH}_{2} \mathrm{Cl}_{2}$ was freshly distilled from powdered $\mathrm{CaH}_{2}$ under $\mathrm{N}_{2}$ prior to use. Flash chromatography was performed using silica gel 60 $\AA$ A (37-70 $\mu \mathrm{m})$. Analytical TLC was carried out utilizing $0.25 \mathrm{~mm}$ precoated plates, silica gel 60 $\mathrm{UV}_{254}$ and spots were visualized by use of UV light or ethanolic phosphomolybdic acid (5\%) followed by heating. NMR samples were dissolved in $\mathrm{CDCl}_{3}$ or $\mathrm{D}_{2} \mathrm{O}$ and run at room temperature; ${ }^{1} \mathrm{H}(400 \mathrm{MHz}),{ }^{13} \mathrm{C}(100 \mathrm{MHz})$ and ${ }^{19} \mathrm{~F}(376 \mathrm{MHz}) \mathrm{NMR}$ spectra were recorded on a $400 \mathrm{MHz}$ spectrometer whereas ${ }^{31} \mathrm{P}(121 \mathrm{MHz}) \mathrm{NMR}$ spectra were recorded on a $300 \mathrm{MHz}$ spectrometer. Chemical shifts for protons are reported using the residual $\mathrm{CHCl}_{3}$ as internal reference $(\delta 7.26)$ or residual $\mathrm{H}_{2} \mathrm{O}\left(\delta\right.$ 4.79) for $\mathrm{D}_{2} \mathrm{O}$, Carbon spectra were referenced to the shift of the ${ }^{13} \mathrm{C}$ signal of $\mathrm{CDCl}_{3}$ ( $\delta$ 77.0). Mass spectra were measured at $70 \mathrm{eV}(\mathrm{EI})$. Melting points are reported as their uncorrected values. Resolution protocol for compound $\mathbf{2}$ is optimized by in terms of yield and ee by DoE, followed by steepest ascent method. ${ }^{1}$

\section{General procedure for preparation of compound 6-8}

Ethyl 2-phenyl-5,6-dihydro-4H-cyclopenta[d] thiazole-4-carboxylate (6) Ethyl 3-bromo-2oxocyclopentanecarboxylate $(3.00 \mathrm{~g}, 12.8 \mathrm{mmol})$ was dissolved in dry EtOH $(10 \mathrm{ml})$ and $\mathrm{PhC}(\mathrm{S}) \mathrm{NH}_{2}(1.78 \mathrm{~g}, 13.0 \mathrm{mmol})$ was added. The resulting mixture was stirred at $\mathrm{rt}$ for $2 \mathrm{~h}$ and then refluxed for 16h. Evaporation to dryness and portioning $\mathrm{CHCl}_{3}(20 \mathrm{ml})$ and aq. $\mathrm{Na}_{2} \mathrm{CO}_{3}(10 \%, 25$ $\mathrm{ml})$, separation and extraction of aq. layer with $\mathrm{CHCl}_{3}(2 \times 10 \mathrm{ml})$. The combined organic extracts were washed with $\mathrm{H}_{2} \mathrm{O}(20 \mathrm{ml})$, brine $(20 \mathrm{ml})$, dried $\left(\mathrm{Na}_{2} \mathrm{SO}_{4}\right)$ and the solvent was removed in vасиио. Purification by flash chromatography (toluene:EtOAc 90:10) yielded $\mathbf{5}$ as a clear oil, $85 \%$.

\footnotetext{
1 Carlson, R.; Design and Optimization in Organic Synthesis, data handling in science and technology, vol III, Elsevier Amsterdam, 1990.
} 
$\mathrm{R}_{f} 0.45$ (toluene:EtOAc 90:10); IR (KBr) $v_{\max }$ 3062, 2980, 2860, 1733, 1529, 1457, 1369, 1333, 1306, 1181, 1031, 1017, 949, 763 and $692 \mathrm{~cm}^{-1}$; ${ }^{1} \mathrm{H}$ NMR $\delta: 1.30$ (t, J=7.2 Hz 3H, $\left.\mathrm{CH}_{2} \underline{\mathrm{CH}}_{3}\right), 2.77-$ $2.83\left(\mathrm{~m}, 1 \mathrm{H}, \mathrm{CH}_{2}\right), 2.90-2.99\left(\mathrm{~m}, 1 \mathrm{H}, \mathrm{CH}_{2}\right), 3.07-3.16\left(\mathrm{~m}, 1 \mathrm{H}, \mathrm{CH}_{2}\right), 4.02-4.07(\mathrm{~m}, 1 \mathrm{H}, \mathrm{CH}), 4.22$ (m, 1H, $\mathrm{CH}_{2}$ ), 4.24 (dq, J=7.2, 14.5, 2H, $\left.\underline{\mathrm{CH}}_{2} \mathrm{CH}_{3}\right), 7.35-7.43$ (m, 3H, ArH), 7.86-7,92 (m, 2H, ArH);

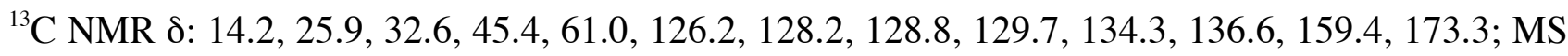
(EI) $(\mathrm{m} / \mathrm{z})$ (rel. intensity) $274.08\left(\mathrm{MH}^{+}, 100 \%\right), 273.12\left(\mathrm{M}^{+}, 90 \%\right), 227.09(79 \%), 200.17(60 \%)$, 199.17 (43\%) and 97.14 (32\%); Calcd. C: 65.91, H: 5.53, N: 5.12; Found C: 65.77, H: 5.63, N: 5.02.

\section{Methyl 2-phenyl-5,6,7,8-tetrahydro-4H-cyclohexa[d]thiazole-4-carboxylate (7)}

Ethyl 3-bromo-2-oxocyclohexanecarboxylate $(3.00 \mathrm{~g}, 17.6 \mathrm{mmol})$ was dissolved in dry EtOH (15 $\mathrm{ml})$ and $\mathrm{PhC}(\mathrm{S}) \mathrm{NH}_{2}(2.45 \mathrm{~g}, 17.9 \mathrm{mmol})$ was added. The resulting mixture was stirred at $\mathrm{rt}$ for $2 \mathrm{~h}$ and then refluxed for 16h. Evaporation to dryness and portioning between $\mathrm{CHCl}_{3}(20 \mathrm{ml})$ and aq. $\mathrm{Na}_{2} \mathrm{CO}_{3}(10 \%, 25 \mathrm{ml})$, separation and extraction of aq. layer with $\mathrm{CHCl}_{3}(2 \times 10 \mathrm{ml})$. The combined organic extracts were washed with $\mathrm{H}_{2} \mathrm{O}(20 \mathrm{ml})$, brine $(20 \mathrm{ml})$, dried $\left(\mathrm{Na}_{2} \mathrm{SO}_{4}\right)$ and the solvent was removed in vacuuo. Purification by flash chromatography (toluene:EtOAc 90:10) yielded 7 as a clear oil, 87\%. $\mathbf{R}_{f} 0.45$ (toluene:EtOAc 90:10). All spectroscopic data were in agreement with previously reported. $^{2}$

\section{Methyl 2-phenyl-5,6,7,8-tetrahydro-4H-cyclohepta[d]thiazole-4-carboxylate (8)}

Synthesized in an analogous method to (6) . Methyl 3-bromo-2-oxocycloheptanecarboxylate, $\mathrm{MeOH}$ used as reaction solvent. Yellow oil; 37\%, $\mathrm{R}_{f} 0.48$ (toluene:EtOAc 90:10); IR (KBr) $v_{\max } 3060,2979$, $2863,1735,1527,1451,1369,1334,1303,1183,1030,1015,946,760$ and $695 \mathrm{~cm}^{-1} ;{ }^{1} \mathrm{H}$ NMR $\delta$ : 1.24-1.34 (m, 2H, $\left.\mathrm{CH}_{2}\right), 1.60-1.69\left(\mathrm{~m}, 2 \mathrm{H}, \mathrm{CH}_{2}\right), 1.85-2.13\left(\mathrm{~m}, 2 \mathrm{H}, \mathrm{CH}_{2}\right), 2.52-2.58\left(\mathrm{~m}, 2 \mathrm{H}, \mathrm{CH}_{2}\right)$, 3.58-3.62 (m, 1H, CH), $3.67\left(\mathrm{~s}, 3 \mathrm{H}, \mathrm{CH}_{3}\right), 7.28-7.35(\mathrm{~m}, 3 \mathrm{H}, \mathrm{ArH}), 7.50-7.56(\mathrm{~m}, 2 \mathrm{H}, \mathrm{ArH}){ }^{13} \mathrm{C}$ NMR $\delta: 19.5,25.9,28.2,28.8,39.2,52.5,127.9,128.7,129.4,131.7,133.2,152.1,168.0,172.9$;

\footnotetext{
${ }^{2}$ Fr. Demande, FR 2035757, 1971
} 
MS (EI) (m/z) $288.10\left(\mathrm{MH}^{+}, 100 \%\right), 287.08\left(\mathrm{M}^{+}, 85 \%\right), 227.09$ (25\%), $200.17(30 \%), 199.17(47 \%)$ and 97.14 (30\%); (rel. intensity) Calcd. C: 66.87, H: 5.96, N: 4.87; Found C: 56.83, H: 5.81, N: 4.79.

\section{General procedure for preparation of Alcohols 9-11}

(S)-(2-phenyl-5,6-dihydro-4H-cyclopenta[d]thiazol-4-yl)methanol (9) Compound 6 (1.00 g, 3.7 mmol) was dissolved in dry THF (5 ml) and added to a slurry of $\mathrm{LiAlH}_{4}(0.14 \mathrm{~g}, 3.7 \mathrm{mmol})$ in THF $(5 \mathrm{ml})$ at $0{ }^{\circ} \mathrm{C}$. The temperature was allowed to rise to $\mathrm{rt}$. and the mixture was stirred o.n. The reaction mixture was recooled to $0^{\circ} \mathrm{C}$ and quenched according to Fieser. ${ }^{3}$ Filtration on Celite, followed by washing of the filter cake with THF $(2 \times 10 \mathrm{ml})$ and evaporation of the combined filtrate gave a crude solid. Purification by flash chromatography $\left(\mathrm{CHCl}_{3}: \mathrm{EtOAc} 7: 3\right)$ yielded the pure compound rac-9, 86\%. Mp: 110.-111.0 ${ }^{\circ} \mathrm{C} ; \mathrm{R}_{f} 0.5$ (EtOAc); HPLC (analytical): Chiracel OD-H column $(4.6 \times 250 \mathrm{~mm})$, hexane: $i$-PrOH 75:25, $0.5 \mathrm{ml} / \mathrm{min}, \mathrm{t}_{\mathrm{R}} 9.5 \mathrm{~min}(R)$ and $14.9 \mathrm{~min}(S)$; Enantiomers separated by HPLC (semi-preparative): Chiracel OD column (20x250 mm), hexane: $i$ PrOH 60:40, $6.0 \mathrm{ml} / \mathrm{min}, 50 \mathrm{mg}$ loading, $\mathrm{t}_{\mathrm{R}} 11.8(R)$ and $19.45 \min (S)$; Mp: 90.8-92.0 ${ }^{\circ} \mathrm{C} ;[\alpha]^{20.4}{ }_{\mathrm{D}}$ $+24.8^{\circ}\left(c 0.99, \mathrm{CHCl}_{3}\right) ; \mathrm{IR}(\mathrm{KBr}) v_{\max } 3338,3063,2941,2856,1531,1499,1456,1428,1310,1228$, 1036, 763, 732 and $691 \mathrm{~cm}^{-1}$; ${ }^{1} \mathrm{H}$ NMR $\delta: 2.14-2.30\left(\mathrm{~m}, 1 \mathrm{H}, \mathrm{CH}_{2}\right), 2.59-2.71\left(\mathrm{~m}, 1 \mathrm{H}, \mathrm{CH}_{2}\right), 2.81$ (brs, $1 \mathrm{H}, \mathrm{OH}) 2.90-3.01\left(\mathrm{~m}, 2 \mathrm{H}, \mathrm{CH}_{2}\right), 3.35-3.48(\mathrm{~m}, 1 \mathrm{H}, \mathrm{CH}), 3.75\left(\mathrm{dd}, J=7.4,10.6 \mathrm{~Hz}, 1 \mathrm{H}, \mathrm{CH}_{2} \mathrm{OH}\right)$, $3.92\left(\mathrm{dd}, J=5.4,10.6 \mathrm{~Hz}, 1 \mathrm{H}, \mathrm{CH}_{2} \mathrm{OH}\right)$, , 7.35-7.48 (m, 3H, ArH), 7.85-7.95 (m, $\left.2 \mathrm{H}, \mathrm{ArH}\right) ;{ }^{13} \mathrm{C} \mathrm{NMR}$

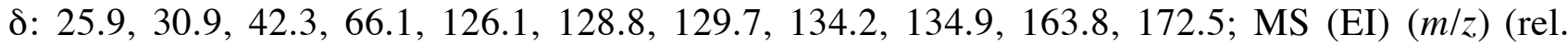
intensity) $232.09\left(\mathrm{MH}^{+}, 47 \%\right), 231.04,\left(\mathrm{M}^{+}, 50 \%\right), 201.12(64 \%), 200.12(100 \%), 121.10(11 \%)$, $104.12(16 \%)$ and 97.09 (57\%) Calcd. C: 67.50, H: 5.66, N: 6.06; Found C: 67.56, H: 5.73, N: 6.05.

(S)-(2-phenyl-4,5,6,7-tetrahydrobenzo[d]thiazol-4-yl)methanol (10) Synthesized in an analogous method to (9). 85\%; Mp: 93.2-94.8 ${ }^{\circ} \mathrm{C} ; . \mathrm{R}_{f} 0.43$ (toluene:EtOAc 80:20); HPLC: (analytical):

${ }^{3}$ Fieser, L. F.; Fieser, M. Reagents for Organic Synthesis; Wiley \& Sons. New York, 1967, $1^{\text {st }}$ ed. 
Chiracel OD-H column (4.6x250 mm), hexane: $i$-PrOH 90:10, $0.5 \mathrm{ml} / \mathrm{min}, \mathrm{t}_{\mathrm{R}} 12.73 \mathrm{~min}(R)$ and $16.81 \mathrm{~min}(S)$; Enantiomers separated by HPLC (semi-preparative): Chiracel OD column (20x250 $\mathrm{mm}$ ), hexane: $i$-PrOH 90:10, $6.0 \mathrm{ml} / \mathrm{min}, 50 \mathrm{mg}$ loading, $\mathrm{t}_{\mathrm{R}} 12.8 \mathrm{~min}(R)$ and $16.9(S) \min ;[\alpha]_{\mathrm{D}}^{20.4}$ $+38.0^{\circ}\left(c 1.3, \mathrm{CHCl}_{3}\right)$; IR $(\mathrm{KBr}) v_{\max } 3365,3062,3009,2938,2865,1723,1667,1605,1542,1459$, 1334, 1043, 761 and $690 \mathrm{~cm}^{-1} ;{ }^{1} \mathrm{H}$ NMR $\delta: 1.39-1.51\left(\mathrm{~m}, 1 \mathrm{H}, \mathrm{CH}_{2}\right), 1.75-1.89\left(\mathrm{~m}, 1 \mathrm{H}, \mathrm{CH}_{2}\right), 1.90-$ $2.10\left(\mathrm{~m}, 2 \mathrm{H}, \mathrm{CH}_{2}\right), 2.57-2.91\left(\mathrm{~m}, 2 \mathrm{H}, \mathrm{CH}_{2}\right), 3.02-3.17(\mathrm{~m}, 1 \mathrm{H}, \mathrm{CH}), 3.69(\mathrm{dd}, J=9.3,10.7 \mathrm{~Hz}, 1 \mathrm{H}$, $\left.\mathrm{CH}_{2} \mathrm{OH}\right), 3.83$ (dd, $\left.J=4.7,10.7 \mathrm{~Hz}, 1 \mathrm{H}, \mathrm{CH}_{2} \mathrm{OH}\right), 4.69$ (brs, $\left.1 \mathrm{H}, \mathrm{OH}\right), 7.38-7.48$ (m, 3H, ArH), 7.85-

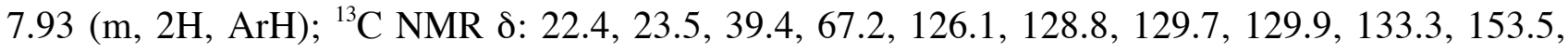
165.2; MS (EI) (m/z) (rel. intensity) $246.11\left(\mathrm{MH}^{+}, 82 \%\right), 245.51\left(\mathrm{M}^{+}, 26 \%\right), 215.12(100 \%), 214.13$ (75\%), 121.09 (28\%), 111.09 (22\%) and 97.09 (17\%) Calcd. C: 68.54, H: 6.16, N: 5.71; Found C: 68.82, H: 6.14, N: 5.64.

(S)-(2-phenyl-5,6,7,8-tetrahydro-4H-cyclohepta[d]thiazol-4-yl)methanol (11) Synthesized in an analogous method to (9). Oil, 73\%, $\mathrm{R}_{f} 0.41$ (toluene:EtOAc 1:1); HPLC (analytical): Chiracel OD-H column $(4.6 \times 250 \mathrm{~mm})$, hexane: $i$ - $\mathrm{PrOH} 90: 10,0.5 \mathrm{ml} / \mathrm{min}, \mathrm{t}_{\mathrm{R}} 13.2 \mathrm{~min}(R)$ and $17.7 \mathrm{~min}(S)$; Enantiomers separated by HPLC (semi-preparative): Chiracel OD column (20x250 mm), hexane: $i$ $\mathrm{PrOH} 80: 20,6.0 \mathrm{ml} / \mathrm{min}, 50 \mathrm{mg}$ loading, $\mathrm{t}_{\mathrm{R}} 14.9 \mathrm{~min}(R)$ and $20.3 \mathrm{~min}(S)[\alpha]^{20.4}{ }_{\mathrm{D}}-55.1^{\circ}(c 4.0$, $\left.\mathrm{CHCl}_{3}\right)$; IR (KBr) $v_{\max } 3326,3049,2936,2858,1528,1454,1425,1308,1223,1078,1031,759$ and $688 \mathrm{~cm}^{-1}$; ${ }^{1} \mathrm{H}$ NMR $\delta: 1.39-1.61\left(\mathrm{~m}, 2 \mathrm{H}, \mathrm{CH}_{2}\right), 1.61-1.83\left(\mathrm{~m}, 2 \mathrm{H}, \mathrm{CH}_{2}\right), 1.92-2.03\left(\mathrm{~m}, 1 \mathrm{H}, \mathrm{CH}_{2}\right), 2.10-$ $2.24\left(\mathrm{~m}, 1 \mathrm{H}, \mathrm{CH}_{2}\right), 2.71-2.84(\mathrm{~m}, 1 \mathrm{H}, \mathrm{CH}), 2.95-3.04(\mathrm{~m}, 1 \mathrm{H}), 3.06$ (brs, $\left.1 \mathrm{H}, \mathrm{OH}\right), 3.95$ (ddd, J=4.2, 9.3, $\left.11.2 \mathrm{~Hz}, 1 \mathrm{H}, \mathrm{CH}_{2} \mathrm{OH}\right), 4.04\left(\mathrm{ddd}, J=4.6,8.5,11.2 \mathrm{~Hz}, 1 \mathrm{H}, \mathrm{CH}_{2} \mathrm{OH}\right), 4.62$ (dd, J=4.6, 9.3, 1H,

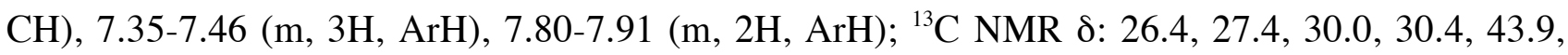
$66.9,125.9,128.8,129.5,133.5,133.8,156.8,162.5$; MS (EI) $(m / z)$ (rel. intensity) 260.10 $\left(\mathrm{MH}^{+}\right.$, 100\%), $259.50\left(\mathrm{M}^{+}, 20 \%\right), 229.11$ (92\%), 228.17 (26\%), $201.14(20 \%), 200.16(15 \%), 121.11(16 \%)$ and 97.10 (14\%) Calcd. C: 69.46, H: 6.61, N: 5.40; Found C: 69.41, H: 6.68, N: 5.35 


\section{General procedure for preparation of compounds 12-14}

\section{(S)-(2-phenyl-5,6-dihydro-4H-cyclopenta[d]thiazol-4-yl)methyl 4-methylbenzenesulfonate (12)}

Compound 9 (1.20 g, $4.9 \mathrm{mmol})$ was dissolved in $\mathrm{CH}_{2} \mathrm{Cl}_{2}(10 \mathrm{ml})$ and pyridine $(10 \mathrm{ml})$ was added. The resulting mixture was cooled to $0{ }^{\circ} \mathrm{C}$ and a solution of $\mathrm{TsCl}(1.39 \mathrm{~g}, 7.3 \mathrm{mmol})$ in $\mathrm{CH}_{2} \mathrm{Cl}_{2}(5 \mathrm{ml})$ was added drop wise over $5 \mathrm{~min}$. The temperature was allowed to rise to rt. and the mixture was stirred o.n. The reaction mixture was poured into aq. $\mathrm{Na}_{2} \mathrm{CO}_{3}(10 \%, 20 \mathrm{ml})$ and the aqueous layer separated and extracted with $\mathrm{CH}_{2} \mathrm{Cl}_{2}(2 \times 20 \mathrm{ml})$. The combined organic layers were washed with $\mathrm{H}_{2} \mathrm{O}$ $(2 \times 30 \mathrm{ml})$, Brine $(30 \mathrm{ml})$, dried $\left(\mathrm{Na}_{2} \mathrm{SO}_{4}\right)$ and evaporated to dryness. Purification by flash chromatography (toluene:EtOAc 9:1) yielded the pure compound 12,78 or $85 \%{ }^{4}, \mathrm{mp} 85.5-87.2{ }^{\circ} \mathrm{C}$; $\mathrm{R}_{f} 0.41$ (toluene:EtOAc 9:1); $[\alpha]^{20.4}+45.6^{\circ}\left(c 0.90, \mathrm{CHCl}_{3}\right)$; IR $(\mathrm{KBr}) v_{\max } 3050,2950,2851,1595$, 1528, 1492, 1454, 1359, 1188, 1176, 1095, 967, 812 and $664 \mathrm{~cm}^{-1} ;{ }^{1} \mathrm{H}$ NMR $\delta: 2.31\left(\mathrm{~s}, 3 \mathrm{H}, \mathrm{ArCH}_{3}\right)$, 2.37-2.48 (m, $\left.1 \mathrm{H}, \mathrm{CH}_{2}\right), 2.66-2.78\left(\mathrm{~m}, 1 \mathrm{H}, \mathrm{CH}_{2}\right), 2.84-3.05\left(\mathrm{~m}, 2 \mathrm{H}, \mathrm{CH}_{2}\right), 3.42-3.52(\mathrm{~m}, 1 \mathrm{H} \mathrm{CH})$, 4.13 (dd, J=7.0, 9.6 Hz, 1H, $\mathrm{CH}_{2} \mathrm{OTs}$ ), 4.42 (dd, J=3.5, 9.6 Hz, 1H, $\mathrm{CH}_{2} \mathrm{OTs}$ ), 7.19-7.26 (m, 2H,

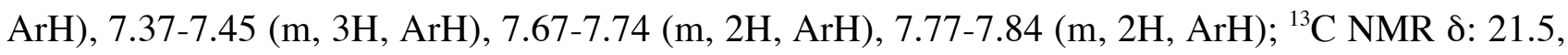
25.6, 31.7, 39.7, 72.3, 126.0, 127.9, 128.4, 129.6, 129.7, 132.7, 134.2, 136.3, 144.6, 160.3, 172.3; MS (EI) (m/z) (rel. intensity) $386.1\left(\mathrm{MH}^{+}, 20 \%\right), 385.1\left(\mathrm{M}^{+}, 17 \%\right), 214.14(25 \%), 213.17(100 \%)$, $200.18(23 \%), 180.22(19 \%), 121.11(10 \%), 110.12(16 \%), 97.12(37 \%), 91.17(41 \%)$ and 83.07 (46\%) Calcd. C: 62.31, H: 4.97, N: 3.63; Found C: 62.29, H: 4.89, N: 3.60.

\section{(S)-(2-phenyl-4,5,6,7-tetrahydrobenzo[d]thiazol-4-yl)methyl 4- methylbenzenesulfonate (13)} Synthesized in an analogous method to (12). Oil, solidifying upon standing, $79 \%$. mp. $77.0-78.1{ }^{\circ} \mathrm{C}$; $\mathrm{R}_{f} 0.45$ (toluene:EtOAc 9:1); $[\alpha]_{\mathrm{D}}^{20.4}+75.6^{\circ}\left(c 2.8, \mathrm{CHCl}_{3}\right) ; \mathrm{IR}(\mathrm{KBr}) v_{\max } 3056,2942,1460,1189$, 1176, 1096, 953, 814, 763, 691 and $666 \mathrm{~cm}^{-1}$; ${ }^{1} \mathrm{H}$ NMR $\delta: 1.69-2.10\left(\mathrm{~m}, 4 \mathrm{H}, \mathrm{CH}_{2}\right), 2.35(\mathrm{~s}, 3 \mathrm{H}$, $\left.\mathrm{ArCH}_{3}\right), 2.75-2.80\left(\mathrm{~m}, 2 \mathrm{H}, \mathrm{CH}_{2}\right), 3.17-3.28(\mathrm{~m}, 1 \mathrm{H}, \mathrm{CH}), 4.24\left(\mathrm{dd}, J=8.2,9.4 \mathrm{~Hz}, 1 \mathrm{H}, \mathrm{CH}_{2} \mathrm{OTs}\right)$, 4.58 (dd, $\left.J=3.6,9.4 \mathrm{~Hz}, 1 \mathrm{H}, \mathrm{CH}_{2} \mathrm{OTs}\right), 7.20-7.29$ (m, 2H, ArH), 7.36-7.44 (m, 3H, ArH), 7.70-7.83

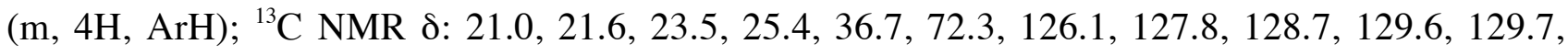

\footnotetext{
${ }^{4}$ The difference in yield depends on which synthetic pathway was followed, scheme $\mathbf{2}$ or $\mathbf{3}$.
} 
131.9, 132.9, 133.7, 144.5, 149.0, 164.7; MS (EI) (m/z) (rel. intensity) 400.84 $\left(\mathrm{MH}^{+}, 35 \%\right), 399.98$ $\left(\mathrm{M}^{+}, 15 \%\right), 354.84$ (41\%), 229.04 (16\%), $227.11(50 \%), 200.96$ (75\%), 155.01 (76\%), 92.12 (70\%), 91.11 (100\%) and 83.03 (49\%) Calcd. C: 63.13, H: 5.30, N: 3.51; Found C: 63.16, H: 5.26, N: 3.47. (S) - (2 - phenyl-5,6,7,8-tetrahydro-4H-cyclohepta[d]thiazol-4-yl)methyl 4methylbenzenesulfonate (14) Synthesized in an analogous method to (12). 71\%, mp 78.6-79.8 ${ }^{\circ} \mathrm{C}$; $\mathrm{R}_{f} 0.44$ (toluene:EtOAc 9:1); $[\alpha]_{\mathrm{D}}^{20.4}+14.0^{\circ}\left(c 0.9, \mathrm{CHCl}_{3}\right)$; IR $(\mathrm{KBr}) v_{\max } 3057,2926,2843,1598$, 1458, 1359, 1189, 1176, 1098, 954, 926, 814 and $666 \mathrm{~cm}^{-1} ;{ }^{1} \mathrm{H}$ NMR $\delta: 1.60-1.88\left(\mathrm{~m}, 4 \mathrm{H}, \mathrm{CH}_{2}\right)$, 1.91-2.09 (m, 2H, $\left.\mathrm{CH}_{2}\right), 2.41\left(\mathrm{~s}, 3 \mathrm{H}, \mathrm{ArCH}_{3}\right), 2.72-2.93\left(\mathrm{~m}, 2 \mathrm{H}, \mathrm{CH}_{2}\right), 3.31-3.50(\mathrm{~m}, 1 \mathrm{H}, \mathrm{CH}), 4.37$ (dd, $J=9.0,9.6 \mathrm{~Hz}, 1 \mathrm{H}, \mathrm{CH}_{2} \mathrm{OTs}$ ), 4.60 (dd, J=4.7, 9.6 Hz, 1H, $\mathrm{CH}_{2} \mathrm{OTs}$ ), 7.29-7.49 (m, 5H, ArH),

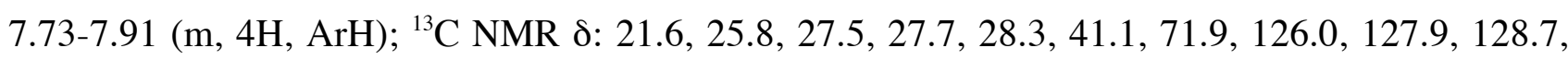
129.4, 129.7, 133.0, 133.7, 134.2, 144.5, 153.2, 162.3; MS (EI) (m/z) (rel. intensity) $414.14\left(\mathrm{MH}^{+}\right.$, 25\%), $413.05\left(\mathrm{M}^{+}, 24 \%\right), 242.16(29 \%), 241.15$ (100\%), 226.2 (21\%), 201.04 (30\%), 155.06 (18\%), $92.17(17 \%)$ and 91.16 (47\%) Calcd. C: 63.89, H: 5.61, N: 3.39; Found C: 63.83, H: 5.55, N: 3.41.

\section{General procedure for preparation of compound 15-17}

(S)-4-((diphenylphosphino)methyl)-2-phenyl-5,6-dihydro-4H-cyclopenta[d]thiazole Borane

adduct (15). Diphenylphospine-borane adduct (0.23 g, $1.1 \mathrm{mmol})$ was dissolved in dry THF (2 ml) and cooled to $-78{ }^{\circ} \mathrm{C}$ under Ar. $n$-BuLi (1.6 M in hexane, $\left.0.71 \mathrm{ml}, 1.15 \mathrm{mmol}\right)$ was added drop wise. The reaction mixture was stirred for $10 \mathrm{~min}$ at $-78{ }^{\circ} \mathrm{C}$, followed by $30 \mathrm{~min}$ at $0{ }^{\circ} \mathrm{C}$. Tosylate 12 (0.301 $\mathrm{g}, 0.75 \mathrm{mmol})$ was added drop wise as a solution in dry DMF $(2 \mathrm{ml})$ and the resulting mixture was stirred at rt. o.n. The reaction mixture was poured into aq. $\mathrm{NaHCO}_{3}(10 \%, 30 \mathrm{ml})$ and extracted with $\mathrm{CH}_{2} \mathrm{Cl}_{2}(3 \times 10 \mathrm{ml})$. The combined organic extracts were washed with $\mathrm{H}_{2} \mathrm{O}(30 \mathrm{ml})$, brine $(30$ $\mathrm{ml})$, dried $\left(\mathrm{MgSO}_{4}\right)$ and evaporated to dryness. Purification by flash chromatography (toluene) gave 15 as clear oil $81 \% \mathrm{R}_{f} 0.61$ (toluene); $[\alpha]^{20.4}+27.5^{\circ}\left(c 0.73, \mathrm{CHCl}_{3}\right)$; IR $(\mathrm{KBr}) v_{\max } 3057,2943$, $2851,2369,1528,1496,1455,1436,1308,1107,1061,1006,762,735$ and $691 \mathrm{~cm}^{-1} ;{ }^{1} \mathrm{H}$ NMR $\delta$ : 0.64-1.57 (m, 3H, $\left.\mathrm{BH}_{3}\right), 2.05-2.18\left(\mathrm{~m}, 1 \mathrm{H}, \mathrm{CH}_{2}\right), 2.26-2.39\left(\mathrm{~m}, 1 \mathrm{H}, \mathrm{CH}_{2}\right), 2.66(\mathrm{ddd}, J=4.1,8.1,13.3$ $\left.\mathrm{Hz}, 1 \mathrm{H}, \mathrm{CH}_{2} \mathrm{P}\right), 2.75-2.93\left(\mathrm{~m}, 2 \mathrm{H}, \mathrm{CH}_{2}\right), 3.24$ (ddd, J=2.6, 13.3, $\left.14.8 \mathrm{~Hz}, 1 \mathrm{H}, \mathrm{CH}_{2} \mathrm{P}\right), 3.40-3.55$ (m, 
1H, CH), 7.36-7.54 (m, 9H, ArH), 7.71-7.78 (m, 2H, ArH), 7.80-7.91 (m, 4H, ArH); ${ }^{13} \mathrm{C}$ NMR $\delta:$ 25.8, $31.3\left(\mathrm{~d}, J_{\mathrm{CP}}=35.1 \mathrm{~Hz},\right), 35.7,36.3,126.1-132.5$ (arom. C), 134.1, 134.5, 165.0; ${ }^{31} \mathrm{P}$ NMR $\delta$ : 15.4; MS (EI) (m/z) (rel. intensity) $414.18\left(\mathrm{MH}^{+}, 35 \%\right), 413.24\left(\mathrm{M}^{+}, 40 \%\right), 412.99(100 \%), 384.31$ (30\%), 371.29 (17\%), 228.29 (31\%), $199.31(26 \%), 183.29(24 \%)$ and 121.23 (16\%) Calcd. C: 72.65; H: 6.10; N: 3.39; Found C: 72.63, H: 6.05, N: 3.30 .

\section{(S)-4-((diphenylphosphino)methyl)-2-phenyl-4,5,6,7-tetrahydrobenzo[d]thiazole Borane}

adduct (16) Synthesized in an analogous method to (15). $83 \%$ or $95 \% .{ }^{4} \mathrm{R}_{f} 0.65$ (toluene); $[\alpha]^{20.4}{ }_{\mathrm{D}}^{-}$ $5.6^{\circ}$ (c 0.82, $\left.\mathrm{CHCl}_{3}\right)$; IR (KBr) $v_{\max } 3058,2930,2858,2374,1541,1462,1437,1107,1062,976$, 912, 763, 734 and $691 \mathrm{~cm}^{-1} ;{ }^{1} \mathrm{H}$ NMR $\delta: 0.64-1.55\left(\mathrm{~m}, 3 \mathrm{H}, \mathrm{BH}_{3}\right), 1.59-1.81\left(\mathrm{~m}, 2 \mathrm{H}, \mathrm{CH}_{2}\right), 1.82-1.96$ (m, 1H, $\left.\mathrm{CH}_{2}\right), 2.11-2.21\left(\mathrm{~m}, 1 \mathrm{H}, \mathrm{CH}_{2}\right), 2.29$ (ddd, $\left.J=7.7,10.6,14.9 \mathrm{~Hz}, 1 \mathrm{H}, \mathrm{CH}_{2} \mathrm{P}\right), 2.73-2.78(\mathrm{~m}$, $\left.2 \mathrm{H}, \mathrm{CH}_{2}\right), 3.23-3.34(\mathrm{~m}, 1 \mathrm{H}, \mathrm{CH}), 3.46\left(\mathrm{ddd}, J=2.2,10.6,14.7 \mathrm{~Hz}, 1 \mathrm{H}, \mathrm{CH}_{2} \mathrm{P}\right), 7.35-7.55(\mathrm{~m}, 9 \mathrm{H}$,

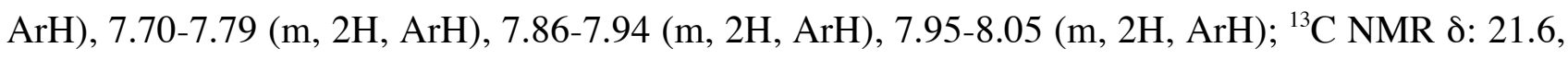
23.7, 29.9, $31.4\left(\mathrm{~d}, J_{\mathrm{CP}}=35.4 \mathrm{~Hz}\right), 33.1,126.1-134.0$ (arom. C), 153.8, 153.9, 164.3; ${ }^{31} \mathrm{P}$ NMR $\delta$ : 16.3; MS (EI) (m/z) (rel. intensity) $428.22\left(\mathrm{MH}^{+}, 20 \%\right), 427.26\left(\mathrm{M}^{+}, 31 \%\right), 426.32(65 \%), 425.31$ (20\%), 336.20 (13\%), 214.17 (13\%), 199.18 (20\%), 183.17 (26\%), 121.12 (35\%), $91.17(44 \%)$, $86.11(61 \%)$ and $84.22(100 \%)$ Calcd. C: 73.07, H: 6.37, N: 3.28; Found C: 72,99, H: 6.29, N: 3.21. (S)-4-((diphenylphosphino)methyl)-2-phenyl-5,6,7,8-tetrahydro-4H-cyclohepta[ $[d]$ thiazole Borane Adduct (17) Synthesized in an analogous method to (15). 73\%. $\mathrm{R}_{f} 0.65$ (toluene); $[\alpha]^{20.4}{ }^{-}$ $33.3^{\circ}\left(c 0.66, \mathrm{CHCl}_{3}\right)$; IR $(\mathrm{KBr}) v_{\max } 3059,2925,2851,2379,1533,1500,1486,1458,1437,1108$, 1061, 910, 763, 735 and $692 \mathrm{~cm}^{-1} ;{ }^{1} \mathrm{H}$ NMR $\delta: 0.62-1.50\left(\mathrm{~m}, 3 \mathrm{H}, \mathrm{BH}_{3}\right), 1.56-1.82\left(\mathrm{~m}, 4 \mathrm{H}, \mathrm{CH}_{2}\right)$, 1.86-2.00 (m, 2H, $\left.\mathrm{CH}_{2}\right), 2.41-2.52\left(\mathrm{~m}, 1 \mathrm{H}, \mathrm{CH}_{2} \mathrm{P}\right), 2.70-2.93\left(\mathrm{~m}, 2 \mathrm{H}, \mathrm{CH}_{2}\right), 3.38-3.56\left(\mathrm{~m}, 2 \mathrm{H}, \mathrm{CH}_{2}\right.$, CH), 7.32-7.48 (m, 9H, ArH), 7.65-7.75 (m, 2H, ArH), 7.76-7.85 (m, 4H, ArH), 7.95-8.05 (m, 2H, ArH); ${ }^{13} \mathrm{C}$ NMR $\delta: 25.2,27.4,28.0,29.4\left(\mathrm{~d}, J_{\mathrm{CP}}=36.5 \mathrm{~Hz},\right), 32.9,37.0,126.0-133.9$ (arom. C),

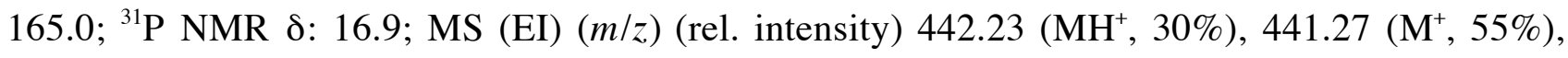
440.27 (95\%), 427.15 (15\%), 398.23 (36\%), 350.23 (100\%), 254.21 (40\%), 213.37 (37\%), 200.23 (43\%), 183. 24 (46\%), 121.19 (38\%) and 91.21 (28\%) Calcd. C: 73.47; H: 6.62; N: 3.17; Found C: 73.45, H: 6.59, N: 3.15. 


\section{General procedure for preparation of compounds 18-20}

Compound $15(0.25 \mathrm{~g}, 0.6 \mathrm{mmol})$ was dissolved in $\mathrm{Et}_{2} \mathrm{NH}(5 \mathrm{ml})$ and the resulting mixture was stirred o.n. at rt. The solvent was removed (in vaccu) and the remaining solid was dissolved in toluene and filtrated through a short plug of silica eluting with toluene, affording the pure product. (S)-4-((diphenylphosphino)methyl)-2-phenyl-5,6-dihydro-4H-cyclopenta[d]thiazole (18) $95 \% \mathrm{R}_{f}$ 0.68 (toluene); $[\alpha]^{20.4}+22.1^{\circ}\left(c 1.05\right.$, EtOAc); IR (KBr) $v_{\max } 3052,2934,2894,2856,1585,1527$, 1497, 1481, 1456, 1434, 1013, 1002, 762, 739, and $696 \mathrm{~cm}^{-1} ;{ }^{1} \mathrm{H}$ NMR $\delta: 1.96-2.18\left(\mathrm{~m}, 2 \mathrm{H}, \mathrm{CH}_{2}\right)$, 2.21-2.56 (m, 3H, $\left.\mathrm{CH}_{2}, \mathrm{CH}\right), 3.00-3.11(\mathrm{~m}, 1 \mathrm{H}, \mathrm{CHP}), 3.12-3.25$ (m, 1H, CHP), 6.90-7.13 (m, 9H,

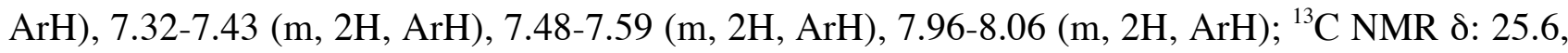
$34.8\left(J_{\mathrm{CP}}=15.2 \mathrm{~Hz}\right), 35.9\left(J_{\mathrm{CP}}=8.5 \mathrm{~Hz}\right), 37.7\left(J_{\mathrm{CP}}=14.5 \mathrm{~Hz}\right), 126.3,128.4-133.6(\mathrm{ArC}), 135.2,138.7$ $\left(J_{\mathrm{CP}}=14.7 \mathrm{~Hz}\right), 140.0\left(J_{\mathrm{CP}}=14.2 \mathrm{~Hz}\right), 166.8\left(J_{\mathrm{CP}}=10.7 \mathrm{~Hz}, \mathrm{CP}\right), 171.7 ;{ }^{31} \mathrm{P}$ NMR $\delta:-19.1$; MS (EI) $(\mathrm{m} / \mathrm{z})$ (rel. intensity) $400.03\left(\mathrm{MH}^{+}, 9 \%\right), 399.06\left(\mathrm{M}^{+}, 22 \%\right), 371.21(23 \%), 214.31(11 \%), 200.32$ (10\%), 166.37 (15\%), 136.37 (13\%), 85.17 (31\%) and 84.30 (100\%) Calcd. C: 75.16; H: 5.55; N: 3.51; Found C: 75.17, H: 5.56, N: 3.50.

(S)-4-((diphenylphosphino)methyl)-2-phenyl-4,5,6,7tetrahydrobenzo[d]thiazole (19)

$93 \%$ or $96 \%,{ }^{4} \mathrm{R}_{f} 0.68$ (toluene); $[\alpha]_{\mathrm{D}}^{20.4}+12.1^{\circ}\left(c 0.95\right.$, EtOAc); IR $(\mathrm{KBr}) v_{\max } 3048,2394,2849$, 1538, 1462, 1434, 974, 762, 739 and $695 \mathrm{~cm}^{-1} ;{ }^{1} \mathrm{H}$ NMR $\delta:\left(1.10-1.35\left(\mathrm{~m}, 1 \mathrm{H}, \mathrm{CH}_{2}\right), 1.42-1.57\right.$ (m, $\left.1 \mathrm{H}, \mathrm{CH}_{2}\right), 1.59-1.76\left(\mathrm{~m}, 1 \mathrm{H}, \mathrm{CH}_{2}\right), 1.89-2.04\left(\mathrm{~m}, 1 \mathrm{H}, \mathrm{CH}_{2}\right), 2.05-2.18\left(\mathrm{~m}, 1 \mathrm{H}, \mathrm{CH}_{2}\right), 2.42,2.19-2.42$ $\left(\mathrm{m}, 2 \mathrm{H}, \mathrm{CH}_{2}\right), 3.00-3.18(\mathrm{~m}, 1 \mathrm{H}), 3.30-3.44(\mathrm{~m}, 1 \mathrm{H}), 6.90-7.20(\mathrm{~m}, 9 \mathrm{H}, \mathrm{ArH}), 7.40-7.53(\mathrm{~m}, 2 \mathrm{H}$,

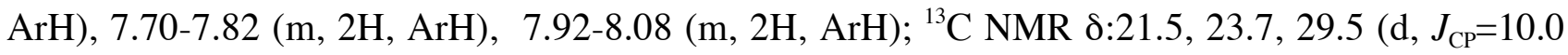
$\mathrm{Hz}), 34.4\left(\mathrm{~d}, J_{\mathrm{CP}}=14.8 \mathrm{~Hz}\right), 35.3\left(\mathrm{~d}, J_{\mathrm{CP}}=16.4 \mathrm{~Hz}\right), 126.5-133.6(\mathrm{ArC}), 134.7,138.9,140.8,155.5$, 164.3; ${ }^{31} \mathrm{P}$ NMR $\delta:-19.1$; MS (EI) ( $\left./ z / z\right)$ (rel. intensity) $414.14\left(\mathrm{MH}^{+}, 26 \%\right), 413.20\left(\mathrm{M}^{+}, 53 \%\right)$, 337.41 (25\%), 336.44 (100\%), 229.78 (29\%), 228.26 (55\%) and 199.21 (20\%) Calcd. C: 75.52; H: 5.85; N: 3.39; Found C: 75.45, H: 5.82, N: 3.38.

(S)-4-((diphenylphosphino)methyl)-2-phenyl-5,6,7,8-tetrahydro-4H-cyclohepta[ $d]$ thiazole (20) $89 \% ; \mathrm{R}_{f} 0.69$ (toluene); $[\alpha]_{\mathrm{D}}^{20.4}-23.4^{\circ}\left(c\right.$ 1.10, EtOAc); IR (KBr) $v_{\max } 3053,3000,2922,2848,1533$, 
1500, 1481, 1459, 1434, 762, 740 and $695 \mathrm{~cm}^{-1} ;{ }^{1} \mathrm{H}$ NMR $\delta: 0.89-1.22\left(\mathrm{~m}, 3 \mathrm{H}, \mathrm{CH}_{2}\right), 1.31-1.63(\mathrm{~m}$, $\left.3 \mathrm{H}, \mathrm{CH}_{2}\right)$ 1.94-2.14 (m, 3H, $\left.\mathrm{CH}_{2}, \mathrm{CH}\right), 2.69-2.84(\mathrm{~m}, 1 \mathrm{H}, \mathrm{CHP}), 3.01-3.21$ (m, 1H, CHP), 6.60-6.83 (m, 9H, ArH), 7.11-7.22 (m, 2H, ArH), 7.23-7.35 (m, 2H, ArH), 7.60-7.68 (m, 2H, ArH); ${ }^{13} \mathrm{C}$ NMR ঠ: 25.3, 27.0, 27.6, $31.5\left(J_{\mathrm{CP}}=11.2 \mathrm{~Hz}\right), 33.0\left(J_{\mathrm{CP}}=14.5 \mathrm{~Hz}\right), 39.3\left(J_{\mathrm{CP}}=15.8 \mathrm{~Hz}\right), 126.0,128.0-128.8$ (ArC), 132.1-134.4 (ArC), $138.8\left(J_{\mathrm{CP}}=15.4 \mathrm{~Hz}\right), 140.5\left(J_{\mathrm{CP}}=15.0 \mathrm{~Hz}\right), 158.6\left(J_{\mathrm{CP}}=6.5 \mathrm{~Hz}\right), 161.4 ;{ }^{31} \mathrm{P}$ NMR 8: -18.8; MS (EI) (m/z) (rel. intensity) $428.12\left(\mathrm{MH}^{+}, 7 \%\right), 427.09\left(\mathrm{M}^{+}, 13 \%\right), 351.27(25 \%)$, 350.43 (100\%), 242.21 (10\%) and 84.76 (12\%) Calcd. C: 75.85; H: 6.13; N: 3.28; Found C: 75.80, $\mathrm{H}: 6.10, \mathrm{~N}: 3.26$.

\section{General procedure for complex formation, compounds 1-3.}

Compound $18(0.140 \mathrm{~g}, 0.35 \mathrm{mmol})$ was dissolved in $10 \mathrm{ml} \mathrm{CH}_{2} \mathrm{Cl}_{2}$ and $\left[\mathrm{IrCl}(\mathrm{COD})_{2}\right]_{2}(0.120 \mathrm{~g}, 0.18$ mmol) was added and the mixture was refluxed 45 min then cooled to $\mathrm{rt} . \mathrm{H}_{2} \mathrm{O}$ was then added (8 ml) followed by addition of $\mathrm{NaBAr}_{\mathrm{F}}(0.40 \mathrm{~g}, 0.42 \mathrm{mmol})$ and the mixture was stirred vigorously for 30 min. The separated organic phase was dried $\left(\mathrm{MgSO}_{4}\right)$ and filtrated before the volatiles were evaporated. The crude product was dissolved in abs. EtOH $(6 \mathrm{ml})$ and $\mathrm{H}_{2} \mathrm{O}$ was added until a precipitate formed. The precipitate was filtered and washed with ice cold aq. EtOH $(80 \%, 2 \mathrm{ml})$. Drying under high vacuum yielded analytically pure Ir- complexes.

Complex 1: $75 \%[\alpha]^{20.4}+25.1^{\circ}\left(c\right.$ 1.5, $\left.\mathrm{CDCl}_{3}\right) ;{ }^{1} \mathrm{H}$ NMR $\delta: 1.22-1.45(\mathrm{~m}, 3 \mathrm{H}), 1.47-1.62(\mathrm{~m}, 1 \mathrm{H})$ 1.73-1.87 (m, 1H), 2.02-2.13 (m, 1H), 2.15-2.26 (m, 2H), 2.31-2.43 (m, 2H), 2.44-2.59 (m, 1H), 2.64-2.75 (m, 1H), 2.94-3.18 (m, 1H) 3.50-3.63 (m, 1H), 4.05-4.20 (m, 1H), 4.56-4.66 (m, 1H), 7.19-7.22 (m, 2H, ArH), 7.41-7.48 (m, 3H, ArH), 7.52-7.69 (m, 9H,ArH, BAr ${ }_{\mathrm{F}}$ ), 7.71-7.77 (m, 2H, ArH), 7.78-8.84 (m, 9H, ArH, BAr $\left.{ }_{\mathrm{F}}\right)$ 7.88-7.93 (m, 2H, ArH); ${ }^{13} \mathrm{C} \mathrm{NMR} \delta: 25.7,25.9,28.2,32.3$ $\left(J_{\mathrm{CP}}=23.5 \mathrm{~Hz}\right), 33.0\left(J_{\mathrm{CP}}=8.9 \mathrm{~Hz}\right), 35.3\left(J_{\mathrm{CP}}=17.0 \mathrm{~Hz}\right), 36.8\left(J_{\mathrm{CP}}=4.0 \mathrm{~Hz}\right), 40.9\left(J_{\mathrm{CP}}=6.0 \mathrm{~Hz}\right), 64.4$, 66.3, 89.4 $\left(J_{\mathrm{CP}}=15.2 \mathrm{~Hz}\right), 97.0\left(J_{\mathrm{CP}}=9.7 \mathrm{~Hz}\right), 117.4\left(\mathrm{~m}, 4 \mathrm{H}, \mathrm{BAr}_{\mathrm{F}}\right), 120.5,123.2,125.9,127.5-132.7$ (ArC), 134.3, 134.5, $134.8\left(\mathrm{~m}, 8 \mathrm{C}, \mathrm{BAr}_{\mathrm{F}}\right), 136.8,161.8\left(\mathrm{q}, J_{\mathrm{CB}}=51.0 \mathrm{~Hz}\right), 162.2\left(J_{\mathrm{CP}}=5.5 \mathrm{~Hz}\right), 176.5$; ${ }^{31} \mathrm{P}$ NMR $\delta: 17.5 ;{ }^{19} \mathrm{~F}$ NMR $\delta:-62.8$; HRMS (FAB+) $\left(\mathrm{M}^{+}\right):$Calcd for $\left[\mathrm{C}_{33} \mathrm{H}_{34} \mathrm{IrNPS}\right]: 700.1779$. Found: 700.1776; Calcd. C: 49.95; H: 2.97; N: 0.90; Found C: 50.21, H: 2.99, N: 1.07. 
Complex 2: 78 or $86 \%{ }^{4}[\alpha]^{20.4}+34.9^{\circ}\left(c 1.05, \mathrm{CDCl}_{3}\right) ;{ }^{1} \mathrm{H}$ NMR $\delta: 1.21-1.49(\mathrm{~m}, 2 \mathrm{H}), 1.77-2.01(\mathrm{~m}$, 4H) $2.02-2.12(\mathrm{~m}, 1 \mathrm{H}), 2.15-2.40(\mathrm{~m}, 3 \mathrm{H}), 2.41-2.55(\mathrm{~m}, 2 \mathrm{H}), 2.65-2.77(\mathrm{~m}, 2 \mathrm{H}), 2.78-2.95(\mathrm{~m}, 3 \mathrm{H})$, 3.25-3.37 (m, 1H), 4.11-4.23 (m, 1H), 4.24-4.31 (m, 1H), 4.52-4.61 (m, 1H), 7.16-7.24 (m, 2H, ArH), 7.40-7.49 (m, 6H, ArH), 7.50-7.53 (m, 4H, BAr $\left.{ }_{\mathrm{F}}\right), 7.54-7.56(\mathrm{~m}, 2 \mathrm{H}, \mathrm{ArH})$, 7.57-7.66 (m, 3H), 7.69-7.74 (m, 8H, BAr $\left.{ }_{\mathrm{F}}\right), 7.96-8.00(\mathrm{~m}, 2 \mathrm{H}, \mathrm{ArH}) ;{ }^{13} \mathrm{C} \mathrm{NMR} \delta: 19.8,23.9,25.6,28.2,30.2\left(J_{\mathrm{CP}}=15.0\right.$ $\mathrm{Hz}), 32.2\left(J_{\mathrm{CP}}=33.9 \mathrm{~Hz}\right), 33.3,37.0\left(J_{\mathrm{CP}}=4.8 \mathrm{~Hz}\right), 37.4\left(J_{\mathrm{CP}}=5.6 \mathrm{~Hz}\right), 64.5,66.3,88.6\left(J_{\mathrm{CP}}=15.3 \mathrm{~Hz}\right)$, $96.3\left(J_{\mathrm{CP}}=8.9 \mathrm{~Hz}\right), 117.4\left(\mathrm{~m}, 4 \mathrm{H}, \mathrm{BAr}_{\mathrm{F}}\right), 120.5,123.2,125.9,127.5-133.4(\mathrm{ArC}), 134.8(\mathrm{~m}, 8 \mathrm{C}$, $\left.\mathrm{BAr}_{\mathrm{F}}\right), 152.2\left(J_{\mathrm{CP}}=3.4 \mathrm{~Hz}\right), 161.5\left(\mathrm{q}, J_{\mathrm{CB}}=49.2 \mathrm{~Hz}\right), 169.2 ;{ }^{31} \mathrm{P}$ NMR $\delta: 11.4 ;{ }^{19} \mathrm{~F}$ NMR $\delta:-63.0$; HRMS (FAB+) $\left(\mathrm{M}^{+}\right)$: Calcd for $\left[\mathrm{C}_{34} \mathrm{H}_{36} \mathrm{IrNPS}\right]:$ 714.1935. Found: 714.1931; Calcd. C: 50.26; H: 3.07; N: 0.89; Found C: 50.53, H: 3.10, N: 0.85.

Complex 3: $63 \%[\alpha]^{20.4}+21.0^{\circ}\left(c\right.$ 1.3, $\left.\mathrm{CDCl}_{3}\right) ;{ }^{1} \mathrm{H}$ NMR $\delta: 1.13-1.52(\mathrm{~m}, 4 \mathrm{H}), 1.72-2.14(\mathrm{~m}, 6 \mathrm{H})$ 2.17-2.33 (m, 2H), 2.34-2.58 (m, 2H), 2.68-3.08 (m, 5H), 3.20-3.32 (m, 1H), 4.33-4.43 (m, 1H), 4.58-4.75 (m, 2H), 7.19-7.31 (m, 3H, ArH), 7.39-7.58 (m, 12H, ArH. BAr $)_{F}, 7.61-7.68(\mathrm{~m}, 2 \mathrm{H}$,

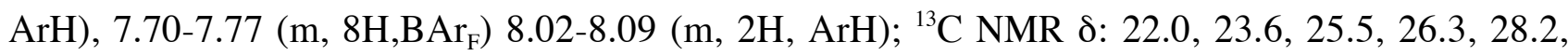
$29.3\left(J_{\mathrm{CP}}=16.8 \mathrm{~Hz}, \mathrm{CP}\right), 31.8\left(J_{\mathrm{CP}}=35.2 \mathrm{~Hz}\right), 33.6\left(J_{\mathrm{CP}}=15.8 \mathrm{~Hz}\right), 37.0,43.6\left(J_{\mathrm{CP}}=3.9 \mathrm{~Hz}\right), 64.7,65.8$, $87.2\left(J_{\mathrm{CP}}=14.9 \mathrm{~Hz}\right), 95.2\left(J_{\mathrm{CP}}=9.9 \mathrm{~Hz}\right), 117.4\left(\mathrm{~m}, 4 \mathrm{H}, \mathrm{BAr}{ }_{\mathrm{F}}\right), 120.5,123.7,125.9,127.5-132.7(\operatorname{ArC})$, $134.8\left(\mathrm{~m}, 8 \mathrm{C}, \mathrm{BAr}_{\mathrm{F}}\right), 136.4,154.0,161.7\left(\mathrm{q}, J_{\mathrm{CB}}=51.0 \mathrm{~Hz}\right), 167.1 ;{ }^{31} \mathrm{P}$ NMR $\delta: 12.2 ;{ }^{19} \mathrm{~F}$ NMR $\delta:-$ 62.8; $\operatorname{HRMS}(\mathrm{FAB}+)\left(\mathrm{M}^{+}\right)$:

Calcd for $\left[\mathrm{C}_{35} \mathrm{H}_{28} \mathrm{IrNPS}\right]:$ 728.2092. Found: 728.2089;

Calcd. C: 50.57; H: 3.17; N: 0.88; Found C: 50.87, H: 3.08, N: 0.96.

Ethyl 2-ammonium-4,5,6,7-tetrahydrobenzo[d]thiazole-4-carboxylate bromide ( $r a c$-25:HBr) To a solution of ethyl 3-bromo-2-oxocyclohexanecarboxylate 5a (43.8 g, $176.0 \mathrm{mmol})$ in abs. EtOH $(200 \mathrm{ml})$ was added thiourea $(14.06 \mathrm{~g}, 184.0 \mathrm{mmol})$ the resulting mixture was stirred at $\mathrm{rt}$. under Ar for $20 \mathrm{~h}$. Evaporation to dryness, followed by trituration (100 $\mathrm{ml}$ abs. EtOH:TBME, 1:1) resulted in a white solid, which was filtered and washed with TBME $(20 \mathrm{ml})$. The filtrate was evaporated to dryness and the triturating procedure repeated (30 $\mathrm{ml}$ abs. EtOH:TBME, 1:1) to give a second crop of crystals, total $51 \mathrm{~g}, 166 \mathrm{mmol}, 94 \% . \mathrm{mp}: 183.6-184.7^{\circ} \mathrm{C}$; IR (KBr) $v_{\max } 3240,3093,2954,2855$, 
2761, 1733, 1623, 1573, 1446, 1185 and $701 \mathrm{~cm}^{-1} ;{ }^{1} \mathrm{H}$ NMR $\left(\mathrm{D}_{2} \mathrm{O}\right) \delta: 1.34(\mathrm{t}, J=7.1 \mathrm{~Hz}, 3 \mathrm{H}$, $\left.\mathrm{CH}_{2} \mathrm{CH}_{3}\right), 1.72-1.87\left(\mathrm{~m}, 1 \mathrm{H}, \mathrm{CH}_{2}\right), 1.87-2.00\left(\mathrm{~m}, 1 \mathrm{H}, \mathrm{CH}_{2}\right), 2.02-2.14\left(\mathrm{~m}, 1 \mathrm{H}, \mathrm{CH}_{2}\right), 2.14-2.26(\mathrm{~m}$, $\left.1 \mathrm{H}, \mathrm{CH}_{2}\right), 2.50-2.66\left(\mathrm{~m}, 2 \mathrm{H}, \mathrm{CH}_{2}\right), 3.74-3.84(\mathrm{~m}, 1 \mathrm{H}, \mathrm{CH}), 4.26\left(\mathrm{t}, J=7.1 \mathrm{~Hz}, 2 \mathrm{H}, \underline{\mathrm{CH}}_{2} \mathrm{CH}_{3}\right) ;{ }^{13} \mathrm{C}$ NMR $\left(\mathrm{D}_{2} \mathrm{O}\right) \delta: 13.5,20.2,22.2,25.3,39.6,63.0,120.1,129.3,168.9,173.8 ; \mathrm{MS}(\mathrm{EI})(\mathrm{m} / \mathrm{z})($ rel. intensity) $227.08\left(\mathrm{MH}^{+}-\mathrm{Br}^{-}, 96 \%\right), 152.16$ (100\%), 119.24 (9\%), 111.15 (15\%). Mol. Wt.: 307.2073 Calcd. $\mathrm{C}_{10} \mathrm{H}_{15} \mathrm{BrN}_{2} \mathrm{O}_{2} \mathrm{~S}: \mathrm{C}, 39.10 ; \mathrm{H}, 4.92 ; \mathrm{N}, 9.12$. Found: C, 39.07; H, 4.96; N, 9.13.

(S)-ethyl 2-amino-4,5,6,7-tetrahydrobenzo[d]thiazole-4-carboxylate $(S)$-25. $40 \mathrm{~g}$ of $\mathrm{rac}$-25: $\mathrm{HBr}$ was dissolved in $\mathrm{H}_{2} \mathrm{O}(400 \mathrm{ml})$ in a E-flask. $20 \mathrm{~g}$ of solid $\mathrm{Na}_{2} \mathrm{CO}_{3}$ was added in portions over $5 \mathrm{~min}$, followed by $\mathrm{CHCl}_{3}(200 \mathrm{ml})$ and the E-flask was put into a warm water bath $\left(50{ }^{\circ} \mathrm{C}\right)$ to ensure complete dissolution of the precipitated freebase. Separation and extraction of the water layer at 50 ${ }^{\circ} \mathrm{C}(2 \times 100 \mathrm{ml} \mathrm{CHCl} 3)$, followed by drying $\left(\mathrm{Na}_{2} \mathrm{SO}_{4}\right)$ evaporation and removal of traces of $\mathrm{CHCl}_{3}$ under oil pump vacuum $\left(1 \times 10^{-2} \mathrm{~mm} \mathrm{Hg}\right)$ gave a white solid, $28.60 \mathrm{~g}, 97 \%$. $\mathrm{R}_{f} 0.48$ (EtOAc); mp 173.3.-174.5 ${ }^{\circ} \mathrm{C}$; IR (KBr) $v_{\max } 3418,3284,3138,2932,2869,1719,1630,1535,1369,11257,1109$, 1045 and $673 \mathrm{~cm}^{-1} ;{ }^{1} \mathrm{H}$ NMR $\delta: 1.24\left(\mathrm{t}, J=7.5 \mathrm{~Hz}, 3 \mathrm{H}, \mathrm{CH}_{2} \mathrm{CH}_{3}\right), 1.69-1.84\left(\mathrm{~m}, 1 \mathrm{H}, \mathrm{CH}_{2}\right), 1.86-2.14$ $\left(\mathrm{m}, 3 \mathrm{H}, \mathrm{CH}_{2}\right), 2.44-2.71\left(\mathrm{~m}, 2 \mathrm{H}, \mathrm{CH}_{2}\right), 3.51-3.67(\mathrm{~m}, 1 \mathrm{H}, \mathrm{CH}), 4.15\left(\mathrm{q}, J=7.5 \mathrm{~Hz}, 2 \mathrm{H}, \mathrm{CH}_{3} \mathrm{CH}_{2}\right)$ $5.29\left(\mathrm{~s}, 2 \mathrm{H}, \mathrm{NH}_{2}\right) ;{ }^{13} \mathrm{C}$ NMR $\delta: 14.2,21.2,22.9,26.7,42.9,60.7,120.4,141.6,165.5,173.8, ; \mathrm{MS}$ (EI) $(\mathrm{m} / \mathrm{z})$ (rel. intensity) $227.08\left(\mathrm{MH}^{+}, 16 \%\right), 226.08\left(\mathrm{M}^{+}, 30 \%\right), 153.11(22 \%), 152.12(32 \%) 85.13$ (58\%), 83.19 (100\%); Calcd. C, 53.08; H, 6.24; N, 12.38. Found C: 52.88; H: 6.19; N: 12.43.

To the flask containing rac-25 (20.00 g, $88.4 \mathrm{mmol})$ was added L-(-)-DBTA monohydrate (33.26 g, $88.4 \mathrm{mmol})$ followed by solvent $\left(\mathrm{H}_{2} \mathrm{O} / \mathrm{MeCN} 20: 80,372 \mathrm{ml}\right)$ and a large magnetic stirring bar. The flask was equipped with a reflux condenser and the resulting thick suspension was heated in an oil bath $\left(90^{\circ} \mathrm{C}\right)$ and kept at reflux until all lumps dissolved and the solution became clear. The heat was then turned off and the mixture was allowed slowly cool down to rt. over $2 \mathrm{~h}$. At the interval of 41 $38^{\circ} \mathrm{C}$ (internal temperature) crystallization starts and the speed of magnetic stirring is increased as the mixture gets thicker. The mixture should be filtered within $2 \mathrm{~h}$ after the crystallization starts, preferably at an internal temperature of ca $25-28{ }^{\circ} \mathrm{C}$. The filter cake was then washed $\left(\mathrm{H}_{2} \mathrm{O}: \mathrm{MeCN}\right.$ 20:80, $50 \mathrm{ml})$ and dried under reduced pressure to give the L-(-)-DBTA-salt of $(S)-2(21.3 \mathrm{~g}, 40 \%)$. 
The recrystallization procedure is then repeated once, using $5 \mathrm{ml}$ solvent $\left(\mathrm{H}_{2} \mathrm{O}: \mathrm{MeCN} 20: 80\right)$ per $\mathrm{g}$ of L-(-)-DBTA:(S)-2. Mp: $139.5-141.7{ }^{\circ} \mathrm{C}$ (decomposition), $[\alpha]^{20.4}{ }_{\mathrm{D}}^{-46.4^{\circ}}$ (c $\left.1.15, \mathrm{MeCN}\right)$ Enantiopure $(S)$-25 was obtained by treatment with $5 \%$ aq $\mathrm{Na}_{2} \mathrm{CO}_{3}$, and extraction with $\mathrm{CHCl}_{3}$ followed by drying $\left(\mathrm{Na}_{2} \mathrm{SO}_{4}\right)$ and evaporation to dryness. +99\% ee, HPLC: Chiracel AS-H column (4.6x250 mm), hexane: $i$-PrOH 90:10, $0.5 \mathrm{ml} / \mathrm{min}, \mathrm{t}_{\mathrm{R}} 20.76 \mathrm{~min}$ minor $(R)$ and $23.12 \mathrm{~min}$ major $(S)$. $[\alpha]^{20.4}-17.7^{\circ}\left(c\right.$ 1.33, $\left.\mathrm{CHCl}_{3}\right) ; \mathrm{Mp}: 100.4-101.8^{\circ} \mathrm{C}$. The unwanted $(R)$-enantiomerically enriched residue can be recycled by racemisation according to the following protocol: Free base of $\mathbf{2 5}$ (10 g, $44.2 \mathrm{mmol})$ was added to a solution of $\mathrm{NaOEt}$ in $\mathrm{EtOH}$, prepared from $\mathrm{Na}(0.05 \mathrm{~g}, 2.2 \mathrm{mmol})$ and absolute EtOH $(100 \mathrm{ml})$. The resulting mixture was then refluxed until a sample (HPLC) indicated complete racemisation (typical $2 \mathrm{~h}$ ) followed by evaporation to dryness. The dry residue is then portioned between $5 \%$ aq. $\mathrm{NaHCO}_{3}(100 \mathrm{ml})$ and $\mathrm{CHCl}_{3}(50 \mathrm{ml})$ Drying and evaporation of organic layer gave racemic $\mathbf{2 5}$, (9.7 $\mathrm{g}, 97 \%)$. This material is usually pure enough for use in the resolution protocol, but decolorization with activated carbon in $90 \%$ aq EtOH (20 ml per $\mathrm{g}$ of crude freebase) gives a higher mass recovery in the resolution procedure.

(S,Z)-ethyl-2-(4-methylphenylsulfonamido)-3-tosyl-2,3,4,5,6,7-hexahydrobenzo[d]thiazole-4carboxylate

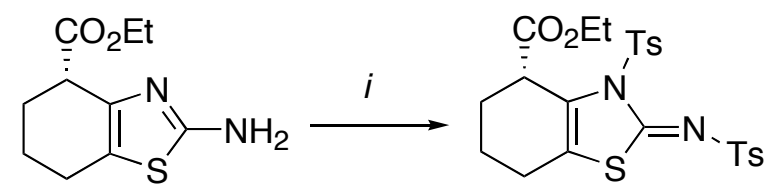

(S)-25

Reagents and conditions: i) 2.1 equiv. $\mathrm{TsCl}$, pyridine, $\mathrm{CH}_{2} \mathrm{Cl}_{2}, 0^{\circ} \mathrm{C}$ to $\mathrm{rt}, 16 \mathrm{~h}, 86 \%$.

To a solution of $(S)-\mathbf{2 5}(0.205 \mathrm{~g}, 0.91 \mathrm{mmol})$ in dry $\mathrm{CH}_{2} \mathrm{Cl}_{2}(3 \mathrm{ml})$ was added pyridine $(2 \mathrm{ml})$ at $0{ }^{\circ} \mathrm{C}$, followed by drop wise addition of tosyl chloride $(0.36 \mathrm{~g}, 1.9 \mathrm{mmol})$ in dry $\mathrm{CH}_{2} \mathrm{Cl}_{2}(2 \mathrm{ml})$. The resulting mixture was stirred at rt. until TLC indicated complete consumption of $(S)-\mathbf{2 5}$ and disappearance of the spot corresponding to monotosylated $25\left(R_{\mathrm{f}} 0.3\right)(16 \mathrm{~h})$. The reaction mixture was then extracted with $10 \%$ aq. $\mathrm{NaHCO}_{3}$, brine, dried $\left(\mathrm{Na}_{2} \mathrm{SO}_{4}\right)$ and evaporated to dryness. 
Purification by flash chromatography (Pentane:EtOAc 6:4) gave the bis tosylate as white crystals, 0.428 g, 88\%. mp 189.0-189.9 ${ }^{\circ} \mathrm{C} ; \mathrm{R}_{f} 0.65$ (hexane:EtOAc 1:1); $[\alpha]^{21.5}{ }_{\mathrm{D}}+7.8^{\circ}\left(c 1.05, \mathrm{CHCl}_{3}\right) ; \mathrm{IR}$ $(\mathrm{KBr}) v_{\max } 3042,2935,2872,1730,1517,1374,1302,1194,1180,1151,1085$ and $726 \mathrm{~cm}^{-1} ;{ }^{1} \mathrm{H}$ NMR $\delta: 1.30\left(\mathrm{t}, J=7.3 \mathrm{~Hz}, 3 \mathrm{H}, \mathrm{CH}_{2} \mathrm{CH}_{3}\right), 1.65-1.79\left(\mathrm{~m}, 1 \mathrm{H}, \mathrm{CH}_{2}\right), 1.85-1.99\left(\mathrm{~m}, 2 \mathrm{H}, \mathrm{CH}_{2}\right), 2.24-$ $2.34\left(\mathrm{~m}, 1 \mathrm{H}, \mathrm{CH}_{2}\right), 2.37\left(\mathrm{~s}, 3 \mathrm{H}, \mathrm{CH}_{3}\right), 2.41\left(\mathrm{~s}, 3 \mathrm{H}, \mathrm{CH}_{3}\right), 2.42-2.57\left(\mathrm{~m}, 2 \mathrm{H}, \mathrm{CH}_{2}\right), 4.18-4.33(\mathrm{~m}, 3 \mathrm{H}$, $\mathrm{CH}_{3} \mathrm{CH}_{2}, \mathrm{CH}$ ), 7.13-7.20 (m, 4H, Ar), 7.58 (m, 2H, Ar), 7.85 (m, 2H. Ar); ${ }^{13} \mathrm{C}$ NMR $\delta: 14.7,18.8$, 21.6, 21.8, 23.9, 26.9, 61.5, 119.2, 126.6, 128.3, 129.094, 129.101, 130.0, 134.1, 138.1, 142.9, 145.9, 164.0, 172.7; MS (EI) $(\mathrm{m} / \mathrm{z})$ (rel. intensity) $535.08\left(\mathrm{M}^{+}, 38 \%\right), 470.08(71 \%), 406.16(22 \%)$, 381.12 (10\%), 379.09 (66\%), 316.15 (24\%), 315.12 (82\%), 305.08 (28\%), 241.20 (25\%), 224.07 (43\%), 155.02 (25\%), 151.12 (30\%), 92.12 (35\%), 91.12 (100\%) and 84.03 (15\%), Calcd. C: 53.91; H, 4.90; N, 5.24; Found C: 53.92, H: 4.72, N: 5.09.

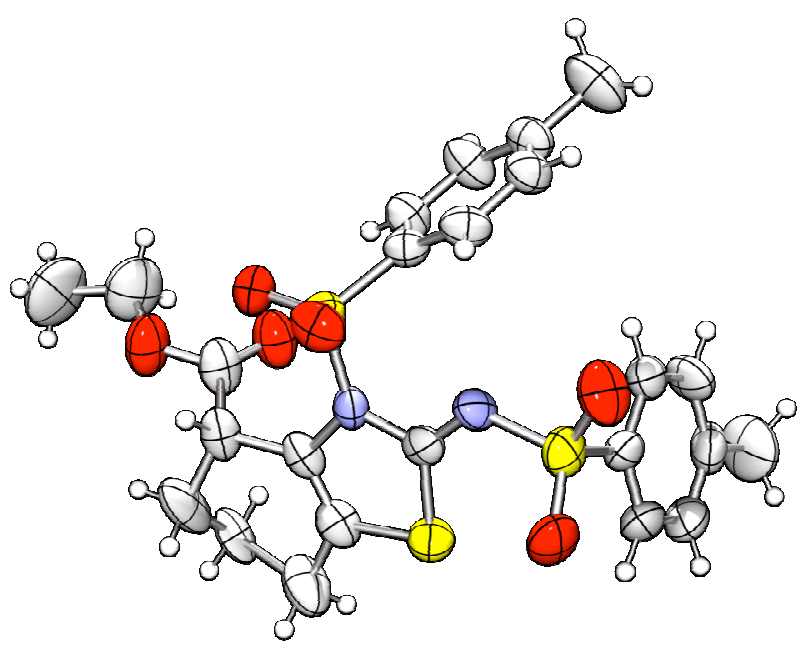

Figure 1: Ortep-drawing of bis tosylated derivative, showing the $(S)$-configuration.

(S)-ethyl 2-bromo-4,5,6,7-tetrahydrobenzo[ $d]$ thiazole-4-carboxylate (26) To a ice cold solution of $(S)-25$ (10.00 g, $44.2 \mathrm{mmol})$ in abs. EtOH (200 ml) 47\% aq. $\mathrm{HBr}(8.52 \mathrm{~g}, 50 \mathrm{mmol})$ was added and the resulting mixture was stirred at rt. for $30 \mathrm{~min}$ and then evaporated to dryness and kept a few hours at oil pump vacuum to remove traces of $\mathrm{H}_{2} \mathrm{O}$ and excess $\mathrm{HBr}$. To the flask containing the resulting dry salt was added a large magnetic stirring bar and dry $\mathrm{MeCN}(150 \mathrm{ml})$ and the resulting suspension was cooled to $0^{\circ} \mathrm{C} . \mathrm{CuBr}_{2}(11.17 \mathrm{~g}, 50.0 \mathrm{mmol})$ was added in one portion and resulting 
deep green mixture was stirred for $10 \mathrm{~min}$ at $0^{\circ} \mathrm{C} . t$-BuONO $(90 \%$ tech. $8.75 \mathrm{ml}, 66.3 \mathrm{mmol})$ was then added drop wise over $5 \mathrm{~min}$. The Resulting mixture was then stirred for $90 \mathrm{~min}$ and poured into a vigorously stirred aq. $\mathrm{Na}_{2} \mathrm{CO}_{3}$ solution $(10 \%, 200 \mathrm{ml})$ containing Celite $535^{\circledR}(5 \mathrm{~g})$ followed by EtOAc $(100 \mathrm{ml})$. The resulting suspension was aged for $30 \mathrm{~min}$ and suction filtered on a sintered funnel containing some Celite $535^{\circledR}$. After separation, the aqueous layer was extracted with EtOAc (3x50 ml) and the combined organic extract was washed with $\mathrm{H}_{2} \mathrm{O}(100 \mathrm{ml})$, brine $(100 \mathrm{ml})$, dried $\left(\mathrm{MgSO}_{4}\right)$ and evaporated to dryness. Purification by flash chromatography (toluene: $\left.\mathrm{Et}_{2} \mathrm{O} 90: 10\right)$ gave 26 clear oil, $10.1 \mathrm{~g}, 79 \% . \mathrm{R}_{f} 0.42$ (toluene/ $\mathrm{Et}_{2} \mathrm{O}$ 80:20); +99\% ee, HPLC, Chiracel AS-H column, (4.6x250 mm), hexane: $i$-PrOH 95:5, $0.5 \mathrm{ml} / \mathrm{min}, \mathrm{t}_{\mathrm{R}} 14.6$ min major $(S)$ and $17.1 \mathrm{~min} \operatorname{minor}(R)$; $[\alpha]_{\mathrm{D}}^{20.4}+11.6^{\circ}\left(c \mathrm{1.55}, \mathrm{CHCl}_{3}\right)$; IR $(\mathrm{KBr}) v_{\max } 2979,2940,2866,1734,1554,1447,1427,1370$, 1335, 1311, 1257, 1237, 1177, 1095, 1025, 995, 857 and $684 \mathrm{~cm}^{-1} ;{ }^{1} \mathrm{H}$ NMR $\delta: 1.28$ (t, J=7.2 Hz, $\left.3 \mathrm{H}, \mathrm{CH}_{2} \mathrm{CH}_{3}\right), 1.80-1.93$ [m, 1H, $\left.\mathrm{CH}_{2}\right), 1.94-2.11\left(\mathrm{~m}, 2 \mathrm{H}, \mathrm{CH}_{2}\right), 2.12-2.21\left(\mathrm{~m}, 1 \mathrm{H}, \mathrm{CH}_{2}\right), 2.64-2.88$ (m, 2H, $\left.\mathrm{CH}_{2}\right), 3.80-3.88(\mathrm{~m}, 1 \mathrm{H}, \mathrm{CH}), 4.21\left(\mathrm{q}, J=7.2 \mathrm{~Hz}, 2 \mathrm{H}, \underline{\mathrm{CH}}_{2} \mathrm{CH}_{3}\right) ;{ }^{13} \mathrm{C}$ NMR $\delta: 14.2,20.7$, 23.2, 26.7, 42.7, 61.0, 132.9, 135.5, 147.3, 172.9; MS (EI) $(m / z)$ (rel. intensity) $291.96\left(\mathrm{MBr}^{81} \mathrm{H}^{+}\right.$, 100\%), $290.97\left(\mathrm{M}^{+} \mathrm{Br}^{81}, 55 \%\right), 290.01\left(\mathrm{MBr}^{79} \mathrm{H}^{+}, 90 \%\right), 289.02\left(\mathrm{M}^{+} \mathrm{Br}^{79}, 46 \%\right), 218.02(50 \%)$, 217.00 (89\%), 216.03 (56\%), 215.03 (83\%), 137.12 (15\%), $136.11(47 \%), 111.10(29 \%), 85.04$ (32\%) and 83.05 (54\%) Calcd. C: 41.39, H: 4.17, N: 4.83; Found C: 41.58, H: 4.11, N: 4.76.

(S)-(2-bromo-4,5,6,7-tetrahydrobenzo[d]thiazol-4-yl)methanol (27) To a solution of 26 (10.00 g, $34.0 \mathrm{mmol})$ in dry THF $(100 \mathrm{ml})$ under $\mathrm{Ar}$ at $-40{ }^{\circ} \mathrm{C}$ was added DIBAL (1.6 $\mathrm{M}$ in toluene, $47.3 \mathrm{ml}$, $75.8 \mathrm{mmol}$ ) drop wise over $10 \mathrm{~min}$. The resulting mixture was stirred at $-40{ }^{\circ} \mathrm{C}$ for $60 \mathrm{~min}$ and quenched with excess of dry $\mathrm{MeOH}(20 \mathrm{ml})$, followed by stirring for $30 \mathrm{~min}$. The resulting cold mixture was poured into a aq. solution of Rochelles salt $(10 \%, 300 \mathrm{ml})$ followed by $\mathrm{CHCl}_{3}(100 \mathrm{ml})$, and stirred until a clear, two-phase mixture was obtained. The phases were separated and the aq. layer extracted with $\mathrm{CHCl}_{3}(2 \times 50 \mathrm{ml})$ the combined organic layers were washed with Rochelles salt solution $(10 \%, 100 \mathrm{ml}), \mathrm{H}_{2} \mathrm{O}(100 \mathrm{ml})$, Brine $(100 \mathrm{ml})$, dried $\left(\mathrm{Na}_{2} \mathrm{SO}_{4}\right)$ and evaporated to dryness. Purification by flash chromatography $\left(\mathrm{CHCl}_{3}\right.$ :EtOAc 9:1 to 4:1) gave 27 as clear oil that solidifies upon standing, $6.1 \mathrm{~g}, 72 \%$. Mp 59.1-60.2 ${ }^{\circ} \mathrm{C} ; \mathrm{R}_{f}$ 0.45, $\left(\mathrm{CHCl}_{3}\right.$ :EtOAc 3:1), HPLC: Chiracel AS-H 
column (4.6x250 mm), hexane: $i$-PrOH 98:2, $0.5 \mathrm{ml} / \mathrm{min}, \mathrm{t}_{\mathrm{R}} 16.6$ major $(S)$ and 18.7 min minor $(R)$ $+99 \% e e ;[\alpha]^{20.4}+42.0^{\circ}\left(c 1.04, \mathrm{CHCl}_{3}\right) ; \mathrm{IR}(\mathrm{KBr}) v_{\max } 3364,2933,2851,1547,1423,1089,1043$, 996 and $995 \mathrm{~cm}^{-1} ;{ }^{1} \mathrm{H}$ NMR $\delta: 1.40-1.56\left(\mathrm{~m}, 1 \mathrm{H}, \mathrm{CH}_{2}\right), 1.70-1.84\left(\mathrm{~m}, 1 \mathrm{H}, \mathrm{CH}_{2}\right), 1.86-2.07(\mathrm{~m}, 2 \mathrm{H}$, $\left.\mathrm{CH}_{2}\right), 2.58-2.80\left(\mathrm{~m}, 2 \mathrm{H}, \mathrm{CH}_{2}\right), 2.91-3.04(\mathrm{~m}, 1 \mathrm{H}, \mathrm{CH}), 3.48$ (brs, $\left.1 \mathrm{H}, \mathrm{OH}\right), 3.69$ (dd, J=7.9, 10.8 Hz,

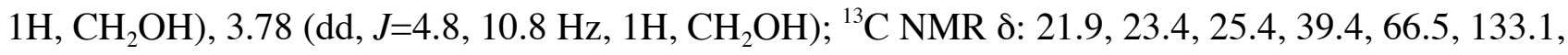
134.1, 152.6; MS (EI) $(m / z)$ (rel. intensity) $250.04\left(\mathrm{MBr}^{81} \mathrm{H}^{+}, 100 \%\right), 248.25\left(\mathrm{MBr}^{79} \mathrm{H}^{+}, 95 \%\right)$, 219.15 (10\%), 218.14 (13\%), 217.05 (8\%), 216.15 (10\%) Calcd. C: 38.72, H: 4.06, N: 5.64; Found C: $38.71, \mathrm{H}: 4.10, \mathrm{~N}: 5.65$.

(S)-(4,5,6,7-tetrahydrobenzo[d]thiazol-4-yl)methanol (28) To a solution of $\mathbf{2 6}(6.50 \mathrm{~g}, 22.4 \mathrm{mmol})$ in dry $\mathrm{THF}(70 \mathrm{ml})$ under $\mathrm{Ar}$ at $-40{ }^{\circ} \mathrm{C}$ was added DIBAL (1.6 $\mathrm{M}$ in toluene, $\left.55.6 \mathrm{ml}, 89.6 \mathrm{mmol}\right)$ drop wise over $10 \mathrm{~min}$, the resulting mixture was then stirred at $-40{ }^{\circ} \mathrm{C}$ for $1 \mathrm{~h}$ and then raised slowly to $0{ }^{\circ} \mathrm{C}$ over $3 \mathrm{~h}$. The reaction mixture was then re-cooled to $-40{ }^{\circ} \mathrm{C}$ and quenched with excess of dry $\mathrm{MeOH}(50 \mathrm{ml})$, followed by stirring for $30 \mathrm{~min}$. The resulting cold mixture was poured into a aq. solution of Rochelles salt $(10 \%, 300 \mathrm{ml})$ followed by $\mathrm{CHCl}_{3}(100 \mathrm{ml})$, and stirred until a clear, twophase mixture was obtained. The phases were separated and the aq. layer extracted with $\mathrm{CHCl}_{3}$ $(2 \times 50 \mathrm{ml})$ and the combined organic layers were washed with Rochelles salt solution $(10 \%, 100 \mathrm{ml})$ $\mathrm{H}_{2} \mathrm{O}(100 \mathrm{ml})$, brine $(100 \mathrm{ml})$, dried $\left(\mathrm{Na}_{2} \mathrm{SO}_{4}\right)$ and evaporated to dryness. Purification by flash chromatography $\left(\mathrm{CHCl}_{3}\right.$ :EtOAc 3:1 to $\left.1: 1\right)$ gave 28 as a slight yellow solid, $3.21 \mathrm{~g}, 85 \%$. mp. 88.7$90.6{ }^{\circ} \mathrm{C} ; \mathrm{R}_{f} 0.21\left(\mathrm{CHCl}_{3}\right.$-EtOAc 3:1); HPLC: Chiracel AS-H column (4.6x250 mm), hexane: $i$-PrOH 90:10, $0.5 \mathrm{ml} / \mathrm{min}, \mathrm{t}_{\mathrm{R}} 17.5$ minor $(R)$ and $20.2 \min$ major $(S),+99 \%$ ee; $[\alpha]^{20.4}{ }_{\mathrm{D}}+28.1^{\circ}(c 1.3$, $\left.\mathrm{CHCl}_{3}\right)$; IR (KBr) $v_{\max } 3244,3048,2950,2927,2857,1546,1417,1370,1049,1005,911$ and 884 $\mathrm{cm}^{-1} ;{ }^{1} \mathrm{H}$ NMR $\delta: 1.36-1.48\left(\mathrm{~m}, 1 \mathrm{H}, \mathrm{CH}_{2}\right), 1.71-1.87\left(\mathrm{~m}, 1 \mathrm{H}, \mathrm{CH}_{2}\right), 1.91-2.11\left(\mathrm{~m}, 2 \mathrm{H}, \mathrm{CH}_{2}\right), 2.68-2.80$ $\left(\mathrm{m}, 1 \mathrm{H}, \mathrm{CH}_{2}\right), 2.81-2.91\left(\mathrm{~m}, 1 \mathrm{H}, \mathrm{CH}_{2}\right), 3.00-3.13(\mathrm{~m}, 1 \mathrm{H}, \mathrm{CH}), 3.66(\mathrm{dd}, J=9.1,10.6 \mathrm{~Hz}, 1 \mathrm{H}$, $\left.\mathrm{CH}_{2} \mathrm{OH}\right), 3.80\left(\mathrm{dd}, J=4.6,10.6 \mathrm{~Hz}, 1 \mathrm{H}, \mathrm{CH}_{2} \mathrm{OH}\right), 4.31$ (brs, $\left.1 \mathrm{H}, \mathrm{OH}\right), 8.59$ (s, $\left.1 \mathrm{H}, \mathrm{ArH}\right) ;{ }^{13} \mathrm{C} \mathrm{NMR} \delta$ : 22.3, 23.4, 25.5, 39.4, 67.1, 129.5, 150.0, 153.1; MS (EI) (m/z) (rel. intensity) $170.11\left(\mathrm{MH}^{+},(100 \%)\right.$, 139.13 (81\%), 138.14 (65\%) and 111.13 (26\%) Calcd. C: 56.77, H: 6.55, N: 8.28; Found C: 56.67, 
H: 6.49, N: 8.31.

(S)-(2-phenyl-4,5,6,7-tetrahydrobenzo[d]thiazol-4-yl)methanol (10) To a solution of 27 (2.0 g, $8.05 \mathrm{mmol})$ in toluene $(20 \mathrm{ml})$ was added $2 \mathrm{~N} \mathrm{Na}_{2} \mathrm{CO}_{3}(8 \mathrm{ml}), \mathrm{PhB}(\mathrm{OH})_{2}(1.46 \mathrm{~g}, 12.0 \mathrm{mmol})$ and $\mathrm{PdCl}_{2}$.dppf.CHCl $\mathrm{CH}_{3}(0.34 \mathrm{~g}, 0.40 \mathrm{mmol})$ and the resulting mixture was degassed and vigorously stirred at $80{ }^{\circ} \mathrm{C}$ under Ar until TLC indicated complete disappearance of 27 (typical $2 \mathrm{~h}$ ). $\mathrm{H}_{2} \mathrm{O}$ (50 $\mathrm{ml})$ and toluene $(30 \mathrm{ml})$ were added followed by $1 \mathrm{~N} \mathrm{NaOH}(10 \mathrm{ml})$. After separation, the aqueous layer was extracted with $\mathrm{CHCl}_{3}(3 \times 50 \mathrm{ml})$ and the combined organic extracts were washed with $\mathrm{H}_{2} \mathrm{O}$ $(100 \mathrm{ml})$, brine $(100 \mathrm{ml})$, dried $\left(\mathrm{MgSO}_{4}\right)$ and evaporated to dryness. Purification by flash chromatography (toluene:EtOAc 90:10) gave $\mathbf{1 0}$ as clear oil, $1.69 \mathrm{~g}, 86 \% . \mathrm{R}_{f} 0.43$ (toulene/EtOAc 80:20); HPLC: Chiracel OD-H column (4.6x250 mm), hexane: $i$-PrOH 90:10, $0.5 \mathrm{ml} / \mathrm{min}, \mathrm{t}_{\mathrm{R}} 12.73$ min minor $(R)$ and 16.81 min major $(S)+99 \% e e ;[\alpha]^{20.4}+38.0^{\circ}\left(c 1.3, \mathrm{CHCl}_{3}\right)$; All spectral and characterization data were in agreement previously synthesized $\mathbf{1 0}$ from the route in scheme 2.

(S)-(4,5,6,7-tetrahydrobenzo[d]thiazol-4-yl)methyl 4-methylbenzenesulfonate (29) Synthesized in an analogous method to (12). 87\%, mp 81.0-82.8 ${ }^{\circ} \mathrm{C} ; \mathrm{R}_{f} 0.41$ (toluene:EtOAc 1:1); $[\alpha]^{20.4}{ }_{\mathrm{D}}-21.4^{\circ}$ $\left(c 0.93, \mathrm{CHCl}_{3}\right) ; \mathrm{IR}(\mathrm{KBr}) v_{\max } 3085,2942,1597,1542,1450,1417,1358,1189,1176,1098,958$, 814 and $666 \mathrm{~cm}^{-1}$; ${ }^{1} \mathrm{H}$ NMR $\delta: ~ 1.70-2.08\left(\mathrm{~m}, 4 \mathrm{H}, \mathrm{CH}_{2}\right), 2.43\left(\mathrm{~s}, 3 \mathrm{H}, \mathrm{ArCH}_{3}\right), 2.73-2.80$ (m, 2H, $\mathrm{CH}_{2}$ ), 3.18-3.28 (m, 1H, CH), $4.12\left(\mathrm{dd}, J=9.0,9.5 \mathrm{~Hz}, 1 \mathrm{H}, \mathrm{CH}_{2} \mathrm{OTs}\right), 4.51(\mathrm{dd}, J=3.8,9.5 \mathrm{~Hz}, 1 \mathrm{H}$, $\left.\mathrm{CH}_{2} \mathrm{OTs}\right), 7.28-7.36(\mathrm{~m}, 2 \mathrm{H}, \mathrm{ArH}), 7.73-7.85$ (m, 2H, ArH), 8.49 (s, 1H, ArH); ${ }^{13} \mathrm{C}$ NMR $\delta: 20.8$, 21.6, 23.3, 25.4, 36.5, 72.1, 127.9, 129.7, 131.5, 132.9, 144.6, 148.7, 149.8;MS (EI) (m/z) (rel. intensity) $324.05\left(\mathrm{MH}^{+}, 15 \%\right), 323.06\left(\mathrm{M}^{+}, 10 \%\right), 229.12(10 \%), 201.00(23 \%), 155.02(23 \%)$, 151.11 (100\%), 136.18 (37\%), 92.22 (31\%), 91.25 (63\%) and 83.29 32\%) Calcd. C: 55.70, H: 5.30, $\mathrm{N}: 4.33$; Found C: 55.75, H: 5.32, N: 4.28.

(S)-4-((dio-tolylphosphino)methyl)-2-phenyl-4,5,6,7-tetrahydrobenzo[ $d]$ thiazole Borane adduct (30) Synthesized in an analogous method to (15). $79 \% . \mathrm{R}_{f} 0.69$ (toluene); $[\alpha]_{\mathrm{D}}^{20.4}-6.98^{\circ}(c 0.86$, $\left.\mathrm{CHCl}_{3}\right)$; IR (KBr) $v_{\max } 3058,2929,2861,2389,1592,1540,1462,1285,1136,1065,911,761,736$ and $691 \mathrm{~cm}^{-1}$; ${ }^{1} \mathrm{H}$ NMR $\delta: 0.65-1.55\left(\mathrm{~m}, 3 \mathrm{H}, \mathrm{BH}_{3}\right), 1.61-1.82\left(\mathrm{~m}, 2 \mathrm{H}, \mathrm{CH}_{2}\right), 1.83-1.97\left(\mathrm{~m}, 1 \mathrm{H}, \mathrm{CH}_{2}\right)$, 2.05-2.19 (m, 4H, $\mathrm{CH}_{2}, \mathrm{ArCH}_{3}$ ), 2.22 (s, 3H, $\mathrm{ArCH}_{3}$ ), 2.30 (ddd, J=7.3, 10.4, $14.5 \mathrm{~Hz}, 1 \mathrm{H}, \mathrm{CH}_{2} \mathrm{P}$ ), 
2.70-2.81 (m, $\left.2 \mathrm{H}, \mathrm{CH}_{2}\right), 3.05-3.17(\mathrm{~m}, 1 \mathrm{H}, \mathrm{CH}), 3.60$ (ddd, J=2.0, 10.4, $\left.14.5 \mathrm{~Hz}, 1 \mathrm{H}, \mathrm{CH}_{2} \mathrm{P}\right), 7.34-$ $7.55(\mathrm{~m}, 9 \mathrm{H}, \mathrm{ArH}), 7.72-7.81(\mathrm{~m}, 2 \mathrm{H}, \mathrm{ArH}), 7.83-7.92(\mathrm{~m}, 1 \mathrm{H}, \mathrm{ArH}), 7.20-8.30(\mathrm{~m}, 1 \mathrm{H}, \operatorname{ArH}) ;{ }^{13} \mathrm{C}$

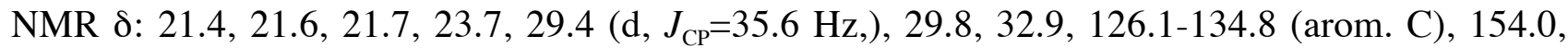

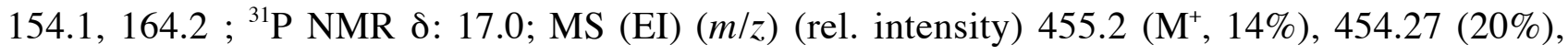
441.17 (25\%), 440.26 (15\%), 350.19 (26\%), 253.17 (16\%), 243.18 (44\%), 242.13 (55\%), 228.18 (100\%), $227.17(68 \%), 213.19(35 \%), 165.19(20 \%), 121.09(14 \%)$ and $91.12(17 \%)$

Calcd. C: 73.85; H: 6.86; N: 3.08; Found C: 73.84, H: 6.80, N: 3.05.

(S)-4-((diphenylphosphino)methyl)-4,5,6,7-tetrahydrobenzo[d]thiazole Borane Adduct (31)

Synthesized in an analogous method to $(\mathbf{1 5}) .75 \% . \mathrm{R}_{f} 0.55$ (toluene: EtOAc $\left.9: 1\right) ;[\alpha]^{20.4}{ }_{\mathrm{D}}+6.7^{\circ}(c$ 0.90, $\mathrm{CHCl}_{3}$ ); IR (KBr) $v_{\max } 3077,3057,3024,3008,2937,6861,2383,1541,1485,1437,1418$, 1108, 1062, 911, 790, 734 and $695 \mathrm{~cm}^{-1} ;{ }^{1} \mathrm{H}$ NMR $\delta: 0.64-1.46\left(\mathrm{~m}, 3 \mathrm{H}, \mathrm{BH}_{3}\right), 1.41-1.59(\mathrm{~m}, 1 \mathrm{H}$, $\left.\mathrm{CH}_{2}\right), 1.61-1.73\left(\mathrm{~m}, 1 \mathrm{H}, \mathrm{CH}_{2}\right), 1.81-1.92\left(\mathrm{~m}, 1 \mathrm{H}, \mathrm{CH}_{2}\right), 2.09-2.18\left(\mathrm{~m}, 1 \mathrm{H} \mathrm{CH}_{2}\right), 2.29$ (ddd, J=8.3, 11.1, 14.1 Hz, 1H, $\left.\mathrm{CH}_{2} \mathrm{P}\right), 2.71-2.76\left(\mathrm{~m}, 2 \mathrm{H}, \mathrm{CH}_{2}\right), 3.23-3.34(\mathrm{~m}, 1 \mathrm{H}, \mathrm{CH}), 3.39$ (ddd, J=2.3, 13.8, 14.1 Hz, 1H, $\left.\mathrm{CH}_{2} \mathrm{P}\right)$, 7.38-7.51 (m, 6H, ArH), 7.71-7.77 (m, 2H, ArH), 7.85-7.92 (m, 2H, ArH), 8.57

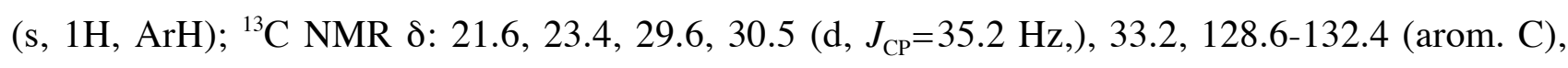

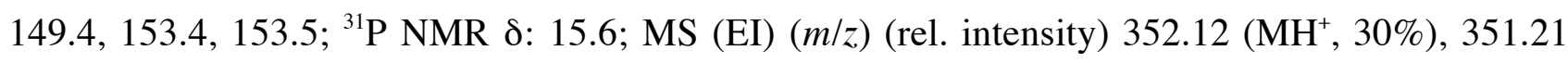
$\left(\mathrm{M}^{+}, 34 \%\right), 350.25(100 \%), 337.20(90 \%), 260.27$ (45\%), $200.32(23 \%), 199.29(38 \%), 183.29$ (34\%), $152.31(65 \%)$ and 121.27 (11\%) Calcd. C: 68.39; H: 6.60; N: 3.99; Found C: 38.35, H: 6.55, $\mathrm{N}: 3.87$.

(S)-4-((dio-tolylphosphino)methyl)-4,5,6,7-tetrahydrobenzo[ $[d]$ thiazole Borane adduct (32) Synthesized in an analogous method to $(\mathbf{1 5}) .76 \% . \mathrm{R}_{f} 0.58$ (toluene: EtOAc 9:1); $[\alpha]_{\mathrm{D}}^{20.4}+28.7^{\circ}(c$ $\left.0.72, \mathrm{CHCl}_{3}\right)$; IR (KBr) $v_{\max } 3056,3009,2932,2859,2385,1591,1540,1451,1418,1284,1134$, 1065, 910 and $737 \mathrm{~cm}^{-1} ;{ }^{1} \mathrm{H}$ NMR $\delta: 0.65-1.55\left(\mathrm{~m}, 3 \mathrm{H}, \mathrm{BH}_{3}\right), 1.57-1.70\left(\mathrm{~m}, 2 \mathrm{H}, \mathrm{CH}_{2}\right), 1.83-2.20(\mathrm{~m}$, 5H, $\mathrm{CH}_{2}, \mathrm{ArCH}_{3}$ ), 2.20 (s, 3H, $\mathrm{ArCH}_{3}$ ), 2.40 (ddd, J=7.0, 10.1, 14.2 Hz, 1H, $\mathrm{CH}_{2} \mathrm{P}$ ), 2.69-2.81 (m, $\left.2 \mathrm{H}, \mathrm{CH}_{2}\right), 2.95-3.12(\mathrm{~m}, 1 \mathrm{H}, \mathrm{CH}), 3.55\left(\mathrm{ddd}, J=1.9,10.1,14.2 \mathrm{~Hz}, 1 \mathrm{H}, \mathrm{CH}_{2} \mathrm{P}\right), 7.20-7.47$ (m, 5H,

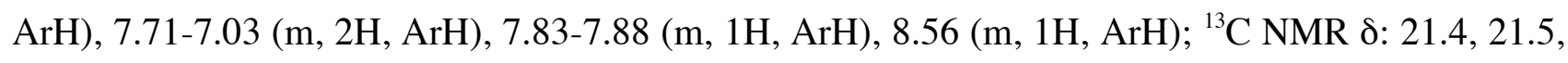
21.6, 23.5, 29.3 (d, $J_{\mathrm{CP}}=35.4 \mathrm{~Hz}$ ), 29.4, 33.2, 125.9-134.4 (arom. C), 149.4, 153.7, 153.8; ${ }^{31} \mathrm{P}$ NMR 
ঠ: 16.6; MS (EI) (m/z) (rel. intensity) $380.43\left(\mathrm{MH}^{+}, 44 \%\right), 379.45\left(\mathrm{M}^{+}, 50 \%\right), 378.52(85 \%), 366.51$ (70\%), 274.24 (23\%), 244.61 (24\%), 243.39 (100\%), 229.38 (85\%), $215.31(81 \%), 213.18873 \%)$, 165.26 (12\%), 153.31 (20\%) and 152.14 (56\%) Calcd. C: 69.66; H: 7.17; N: 3.69; Found C: 69.61, $\mathrm{H}: 7.14, \mathrm{~N}: 3.65$.

(S)-4-((dio-tolylphosphino)methyl)-2-phenyl-4,5,6,7-tetrahydrobenzo[d]thiazole $(33)$ Synthesized in an analogous method to (18). 93\%. $\mathrm{R}_{f} 0.70$ (toluene); $[\alpha]^{20.4}{ }_{\mathrm{D}}+10.8^{\circ}(c 0.89$, EtOAc); IR $(\mathrm{KBr}) v_{\max } 3056,3004,2935,2856,2279,1537,1462,1451,1272,975,750$ and $690 \mathrm{~cm}^{-1} ;{ }^{1} \mathrm{H}$ NMR $\delta:\left(1.11-1.37\left(\mathrm{~m}, 1 \mathrm{H}, \mathrm{CH}_{2}\right), 1.42-1.59\left(\mathrm{~m}, 1 \mathrm{H}, \mathrm{CH}_{2}\right), 1.64-1.80\left(\mathrm{~m}, 1 \mathrm{H}, \mathrm{CH}_{2}\right), 1.83-2.09(\mathrm{~m}\right.$, $\left.2 \mathrm{H}, \mathrm{CH}_{2}\right), 2.19-2.36\left(\mathrm{~m}, 2 \mathrm{H}, \mathrm{CH}_{2}\right), 2.42\left(\mathrm{~s}, 3 \mathrm{H}, \mathrm{ArCH}_{3}\right), 2.51\left(\mathrm{~s}, 3 \mathrm{H}, \mathrm{ArCH}_{3}\right), 3.00-3.17(\mathrm{~m}, 1 \mathrm{H}$, $\mathrm{CH}), 3.27-3.39(\mathrm{~m}, 1 \mathrm{H}), 6.82-7.30(\mathrm{~m}, 10 \mathrm{H}, \mathrm{ArH}), 7.98-8.12(\mathrm{~m}, 3 \mathrm{H}, \mathrm{ArH}) ;{ }^{13} \mathrm{C}$ NMR $\delta: 21.2,21.5$, 21.6, 23.8, $29.5\left(\mathrm{~d}, J_{\mathrm{CP}}=11.0 \mathrm{~Hz}\right), 33.0\left(\mathrm{~d}, J_{\mathrm{CP}}=12.9 \mathrm{~Hz}\right), 35.3\left(\mathrm{~d}, J_{\mathrm{CP}}=16.6 \mathrm{~Hz}\right), 126.5-132.4(\mathrm{ArC})$, 134.8, 136.8, 138.4, 141.8, 141.9, 155.7, 164.4; ${ }^{31} \mathrm{P}$ NMR $\delta:-42.3 ;$ MS (EI) $(m / z)$ (rel. intensity) $442.22\left(\mathrm{MH}^{+}, 20 \%\right), 441.26\left(\mathrm{M}^{+}, 37 \%\right), 351.3(12 \%), 350.27(25 \%), 244.94(27 \%), 243.43(67 \%)$, 228.64 (100\%), 226.99 (38\%), and 213.11 (15\%); Calcd. C: 76.16; H: 6.39; N: 3.17; Found C: 76.12, H: 6.35, N: 5.16 .

(S)-4-((diphenylphosphino)methyl)-4,5,6,7-tetrahydrobenzo[d] thiazole (34) Synthesized in an analogous method to (18). $91 \%$. $\mathrm{R}_{f} 0.62$ (toluene) $[\alpha]_{\mathrm{D}}^{20.4}+13.1^{\circ}\left(c 0.93\right.$, EtOAc); IR $(\mathrm{KBr}) v_{\max }$ 3058, 2933, 2847, 1534, 1480, 1433, 1418, 885, 739 and $697 \mathrm{~cm}^{-1} ;{ }^{1} \mathrm{H}$ NMR $\delta:(1.09-1.29(\mathrm{~m}, 1 \mathrm{H}$, $\left.\mathrm{CH}_{2}\right), 1.36-1.51\left(\mathrm{~m}, 1 \mathrm{H}, \mathrm{CH}_{2}\right), 1.53-1.69\left(\mathrm{~m}, 1 \mathrm{H}, \mathrm{CH}_{2}\right), 1.87-2.07\left(\mathrm{~m}, 2 \mathrm{H}, \mathrm{CH}_{2}\right), 2.14-2.37(\mathrm{~m}, 2 \mathrm{H}$, $\left.\mathrm{CH}_{2}\right), 2.95-3.14(\mathrm{~m}, 1 \mathrm{H}), 3.31-3.45(\mathrm{~m}, 1 \mathrm{H}), 6.92-7.15(\mathrm{~m}, 6 \mathrm{H}, \mathrm{ArH}), 7.34-7.48(\mathrm{~m}, 2 \mathrm{H}, \mathrm{ArH})$, 7.65$7.78(\mathrm{~m}, 2 \mathrm{H}, \mathrm{ArH}) 8.13(\mathrm{~s}, 1 \mathrm{H} \mathrm{ArH}) ;{ }^{13} \mathrm{C} \mathrm{NMR} \delta: 21.6,23.5,29.4\left(\mathrm{~d}, J_{\mathrm{CP}}=11.0 \mathrm{~Hz}\right), 34.5\left(\mathrm{~d}, J_{\mathrm{CP}}=14.4\right.$ $\mathrm{Hz}), 35.1\left(\mathrm{~d}, J_{\mathrm{CP}}=16.1 \mathrm{~Hz}\right), 128.4-133.6(\mathrm{ArC}), 138.7,140.8,148.9,155.1 ;{ }^{31} \mathrm{P}$ NMR $\delta:-19.7 ; \mathrm{MS}$ (EI) $(\mathrm{m} / \mathrm{z})$ (rel. intensity) $338.15\left(\mathrm{MH}^{+}, 26 \%\right), 337.11\left(\mathrm{M}^{+}, 40 \%\right), 260.17(36 \%), 200.22(30 \%)$, 199.25 (100\%), 183.28 (67\%), 152.27 (95\%) and 121.35 (45\%) Calcd. C: 71.19; H: 5.97; N: 4.15; Found C: 71.20; H: 6.02; N: 4.13. 
(S)-4-((dio-tolylphosphino)methyl)-4,5,6,7-tetrahydrobenzo[ $d]$ thiazole (35) Synthesized in an analogous method to (18). $92 \%$. $\mathrm{R}_{f} 0.65$ (toluene); $[\alpha]^{20.4}+11.4^{\circ}\left(c 0.90\right.$, EtOAc); $\mathrm{IR}(\mathrm{KBr}) v_{\max }$ $3055,3004,2936,2857,2279,1589,1539,1469,1451,1418,891,749$ and $719 \mathrm{~cm}^{-1} ;{ }^{1} \mathrm{H}$ NMR $\delta:$ (1.16-1.33 (m, 1H, $\left.\mathrm{CH}_{2}\right), 1.39-1.54\left(\mathrm{~m}, 1 \mathrm{H}, \mathrm{CH}_{2}\right), 1.62-1.86\left(\mathrm{~m}, 1 \mathrm{H}, \mathrm{CH}_{2}\right), 1.91-2.05\left(\mathrm{~m}, 2 \mathrm{H}, \mathrm{CH}_{2}\right)$, 2.15-2.34 (m, 2H, $\left.\mathrm{CH}_{2}\right), 2.39$ (s, 3H, $\left.\mathrm{ArCH}_{3}\right), 2.48$ (s, 3H, $\left.\mathrm{ArCH}_{3}\right), 2.97-3.13$ (m, 1H, CH), 3.22-3.34 $(\mathrm{m}, 1 \mathrm{H}), 6.81-7.26(\mathrm{~m}, 7 \mathrm{H}, \mathrm{ArH}), 7.97-8.06(\mathrm{~m}, 1 \mathrm{H}, \mathrm{ArH}), 8.13\left(\mathrm{~s}, 1 \mathrm{H}\right.$ ArH) ${ }^{13} \mathrm{C}$ NMR $\delta: 21.0,21.3$, 21.4, 23.5, $29.3\left(\mathrm{~d}, J_{\mathrm{CP}}=9.6 \mathrm{~Hz}\right), 33.0\left(\mathrm{~d}, J_{\mathrm{CP}}=12.9 \mathrm{~Hz}\right), 34.9\left(\mathrm{~d}, J_{\mathrm{CP}}=16.8 \mathrm{~Hz}\right), 126.4-132.1(\mathrm{ArC})$, 136.6, 138.3 141.7, 142.7, 149.0, 155.2; ${ }^{31} \mathrm{P}$ NMR $\delta:-40.8$; MS (EI) (m/z) (rel. intensity) $365.03\left(\mathrm{M}^{+}\right.$, 10\%), 166.38 815\%), 152.27 (28\%), 84.56 (100\%). Calcd. C: 72.30; H: 6.62; N: 3.83; Found C: 72.25; H: 6.53; N: 3.79 .

All complexes were synthesized in an analogous method to (1).

Complex 36: 81\%. $[\alpha]^{20.4}+30.8^{\circ}\left(c 0.95, \mathrm{CDCl}_{3}\right)$; ${ }^{1} \mathrm{H}$ NMR $\delta: 1.17-1.45(\mathrm{~m}, 3 \mathrm{H})$ 1.76-1.96 (m, 4H), 1.98-2.12 (m, 4H), 2.15-2.37 (m, 4H), 2.38-2.53 (m, 2H), 2.63-2.94 (m, 6H), 3.22-3.35 (m, 1H), 4.10-4.28 (m, 2H), 4.60-4.71 (m, 1H), 6.60-6.75 (m, 1H, ArH), 7.11-7.19 (m, 1H, ArH), 7.30-7.51 (m, 5H, ArH), 7.52-7.55 (m, 4H, BAr $\mathrm{F}_{\mathrm{F}}$, 7.58-7.66 (m, 2H, ArH), 7.67-7.71 (m, 1H, ArH), 7.72-7.76 $\left(\mathrm{m}, 8 \mathrm{H}, \mathrm{BAr}_{\mathrm{F}}\right), 8.12-8.20(\mathrm{~m}, 2 \mathrm{H}, \mathrm{ArH}), 8.35-8.46(\mathrm{~m}, 1 \mathrm{H}, \mathrm{ArH}) ;{ }^{13} \mathrm{C} \mathrm{NMR} \delta: 20.0,22.6,24.0,25.2$, $27.7,30.7\left(J_{\mathrm{CP}}=15.9 \mathrm{~Hz}\right), 31.8,32.2,33.9,36.7\left(J_{\mathrm{CP}}=6.2 \mathrm{~Hz}\right), 37.4,66.0,66.5,86.9\left(J_{\mathrm{CP}}=16.5 \mathrm{~Hz}\right)$, $95.5\left(J_{\mathrm{CP}}=8.9 \mathrm{~Hz}\right), 117.4\left(\mathrm{~m}, 4 \mathrm{C}, \mathrm{BAr}_{\mathrm{F}}\right), 120.5,123.2,125.9-133.5(\mathrm{ArC}), 134.8\left(\mathrm{~m}, 8 \mathrm{H}, \mathrm{BAr}_{\mathrm{F}}\right)$, 140.0, 142.1, 152.3, $161.7\left(J_{\mathrm{CB}}=50.1 \mathrm{~Hz}\right), 169.5 ;{ }^{31} \mathrm{P}$ NMR $\delta: 12.2 ;{ }^{19} \mathrm{~F}$ NMR $\delta:-62.8 ;$ HRMS $(\mathrm{FAB}+)\left(\mathrm{M}^{+}\right)$: Calcd for $\left[\mathrm{C}_{36} \mathrm{H}_{40}\right.$ IrNPS]: 742.2248. Found: 742.2240; Calcd. C: 50.88; H: 3.27; N: 0.87; Found C: 50.75, H: 3.30, N: 0.98.

Complex 37: 72\%. $[\alpha]_{\mathrm{D}}^{20.4}-7.1^{\circ}\left(c\right.$ 1.42, $\left.\mathrm{CDCl}_{3}\right) ;{ }^{1} \mathrm{H}$ NMR $\delta: 1.44-1.57(\mathrm{~m}, 1 \mathrm{H}), 1.64-1.82(\mathrm{~m}, 2 \mathrm{H})$ 1.86-2.01 (m, 3H), 2.13-2.41 (m, 5H), 2.46-2.57 (m, 2H), 2.63-2.77 (m, 3H), 2.85-2.95 (m, 1H), 3.67-3.77 (m, 1H), 3.78-3.88 (m, 1H), 4.61-4.74 (m, 2H), 7.14-7.21 (m, 2H, ArH), 7.37-7.49 (m, 6H, ArH), 7.50-7.53 (m, 4H, BAr $\left.{ }_{F}\right), 7.62-7.69(\mathrm{~m}, 2 \mathrm{H}, \mathrm{ArH}), 7.70-7.74\left(\mathrm{~m}, 8 \mathrm{H}, \mathrm{BAr}_{\mathrm{F}}\right), 8.63(\mathrm{~s}, 1 \mathrm{H}$,

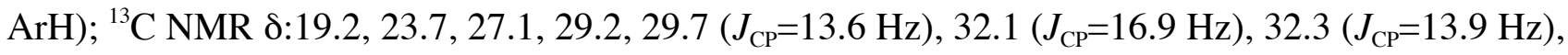
$35.7\left(J_{\mathrm{CP}}=4.4 \mathrm{~Hz}\right), 37.0\left(J_{\mathrm{CP}}=6.4 \mathrm{~Hz}\right), 64.2,67.0,93.8\left(J_{\mathrm{CP}}=14.1 \mathrm{~Hz}\right), 94.4\left(J_{\mathrm{CP}}=10.8 \mathrm{~Hz}\right), 117.5(\mathrm{~m}$, 
4H, BAr $\left.{ }_{\mathrm{F}}\right), 120.5,123.2,125.9,127.5-132.4(\mathrm{ArC}), 133.9,134.6,134.8\left(\mathrm{~m}, 8 \mathrm{C}, \mathrm{BAr}_{\mathrm{F}}\right), 151.4$ $\left(J_{\mathrm{CP}}=4.9 \mathrm{~Hz}\right), 152.9,161.7\left(\mathrm{q}, J_{\mathrm{CB}}=49.8 \mathrm{~Hz}\right) ;{ }^{31} \mathrm{P}$ NMR $\delta: 13.0 ;{ }^{19} \mathrm{~F}$ NMR $\delta:-63.1 ; \mathrm{HRMS}(\mathrm{FAB}+)$ $\left(\mathrm{M}^{+}\right)$: Calcd for $\left[\mathrm{C}_{28} \mathrm{H}_{32} \mathrm{IrNPS}\right]:$ 638.1622. Found: 638.1622; Calcd. C: 48.01; H: 2.95; N: 0.93; Found C: 47.76, H: 3.05, N: 1.10.

Complex 38: $68 \%$. $[\alpha]^{20.4}-31.8^{\circ}\left(c 1.07, \mathrm{CDCl}_{3}\right) ;{ }^{1} \mathrm{H}$ NMR $\delta: 1.44-1.57(\mathrm{~m}, 1 \mathrm{H}), 1.64-1.82(\mathrm{~m}, 2 \mathrm{H})$ 1.84-1.99 (m, 3H), $2.01(\mathrm{~s}, 3 \mathrm{H}), 2.111-2.40(\mathrm{~m}, 8 \mathrm{H}), 2.47-2.59(\mathrm{~m}, 2 \mathrm{H}), 2.62-2.76(\mathrm{~m}, 3 \mathrm{H}), 2.85-$ $2.96(\mathrm{~m}, 1 \mathrm{H}), 3.65-3.75(\mathrm{~m}, 1 \mathrm{H}), 3.77-3.86(\mathrm{~m}, 1 \mathrm{H}), 4.32-4.46(\mathrm{~m}, 1 \mathrm{H})$ 4.61-4.74 (m, 1H), 6.61-6.79 (m, 1H, ArH), 7.11-7.19 (m, 1H, ArH), 7.30-7.51 (m, 3H, ArH), 7.52-7.55 (m, 4H, BAr $\left.{ }_{\mathrm{F}}\right)$, 7.58-7.66 $(\mathrm{m}, 2 \mathrm{H}, \mathrm{ArH}), 7.72-7.76\left(\mathrm{~m}, 8 \mathrm{H}, \mathrm{BAr}_{\mathrm{F}}\right), 8.36-8.47(\mathrm{~m}, 1 \mathrm{H}, \mathrm{ArH}) 8.72(\mathrm{~s}, 1 \mathrm{H}, \mathrm{ArH}) ;{ }^{13} \mathrm{C} \mathrm{NMR} \delta$ : $19.4,22.6\left(J_{\mathrm{CP}}=6.1, \mathrm{~Hz}\right), 22.7,23.7,26.3,28.3,29.8\left(J_{\mathrm{CP}}=16.9 \mathrm{~Hz}\right), 31.0,31.1,33.4,36.3\left(J_{\mathrm{CP}}=5.9\right.$ $\mathrm{Hz}), 66.5,67.1,91.6\left(J_{\mathrm{CP}}=14.9 \mathrm{~Hz}\right), 93.5\left(J_{\mathrm{CP}}=10.4 \mathrm{~Hz}\right), 117.4\left(\mathrm{~m}, 4 \mathrm{C}, \mathrm{BAr}_{\mathrm{F}}\right), 120.5,123.2,125.9-$ $133.4(\mathrm{ArC}), 134.8\left(\mathrm{~m}, 8 \mathrm{H}, \mathrm{BAr}_{\mathrm{F}}\right), 142.1,151.4,152.9,161.6\left(J_{\mathrm{CB}}=49.7 \mathrm{~Hz}\right) ;{ }^{31} \mathrm{P} \mathrm{NMR} \delta: 14.8 ;{ }^{19} \mathrm{~F}$ NMR $\delta:-62.8 ;$ HRMS (FAB+) $\left(\mathrm{M}^{+}\right)$: Calcd for $\left[\mathrm{C}_{30} \mathrm{H}_{36} \mathrm{IrNPS}\right]:$ 666.1935. Found: 666.1934; Calcd. C: 48.70; H: 3.16; N: 0.92; Found C: 48.97, H: 3.23, N: 1.03.

\section{General procedure for hydrogenation reactions.}

A vial was charged with substrate $(0.5 \mathrm{mmol})$ and catalyst $(0.5-1 \mathrm{~mol} \%)$ and $2.0 \mathrm{ml}$ anhydrous $\mathrm{CH}_{2} \mathrm{Cl}_{2}$ was added. The vial was placed in a high-pressure equipment which was purged three times with Ar before it was pressurized to 50 bars and held at this pressure for two hours. The pressure was released and the solvent was evaporated off. $1.5 \mathrm{ml}$ of $\mathrm{Et}_{2} \mathrm{O}$ :pentane (1:1) was added and the solution was filtrated through a short plug of silica. The solvent was evaporated and the enantiomeric excesses were determined by GC (G-TA) or HPLC and conversions were determined by ${ }^{1} \mathrm{H}$ NMR. 


\section{Separation details}

Retention time for enantiomers and starting materials follows below: 


\begin{tabular}{|c|c|c|c|c|c|c|}
\hline & Substrate & Substr & ate $T_{R}(\min )$ & $\mathrm{T}_{\mathrm{R} 1}(\min )$ & $\mathrm{T}_{\mathrm{R} 2}(\mathrm{~min})$ & Separation Method \\
\hline 21 & 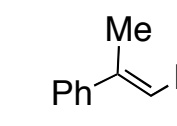 & & 24.5 & $13.3(R)$ minor & $21.8(S)$ major $^{\mathrm{a}}$ & $\begin{array}{l}\text { HPLC (chiracel OJ-H } \\
\text { column, } 254 \mathrm{~nm}, 20^{\circ} \mathrm{C}, n- \\
\text { hexane: } i-\mathrm{PrOH} 99 / 1\end{array}$ \\
\hline 22 & & Me & 43.1 & $37.4(S)$ major $^{\mathrm{a}}$ & $37.6(R)$ minor & $\begin{array}{l}\mathrm{GC}-\mathrm{MS}\left(\mathrm{G}-\mathrm{TA}, 60^{\circ} \mathrm{C}\right. \\
30 \mathrm{~min}, 5^{\circ} \mathrm{C} / \mathrm{min}, 75^{\circ} \mathrm{C} \\
100 \mathrm{kPa})\end{array}$ \\
\hline 39 & & & 18.1 & $8.1(S)$ major $^{b}$ & $8.3(R)$ minor & $\begin{array}{l}\mathrm{GC}-\mathrm{MS}\left(\mathrm{G}-\mathrm{TA}, 60^{\circ} \mathrm{C}, 30\right. \\
\mathrm{min}, 5^{\circ} \mathrm{C} / \mathrm{min}, 175^{\circ} \mathrm{C} \\
100 \mathrm{kPa})\end{array}$ \\
\hline 40 & & & 48.8 & $46.4(R)$ major $^{\mathrm{C}}$ & $46.9(S)$ minor & $\begin{array}{l}\mathrm{GC}-\mathrm{MS}\left(\mathrm{G}-\mathrm{TA}, 60^{\circ} \mathrm{C}\right. \\
30 \mathrm{~min}, 3^{\circ} \mathrm{C} / \mathrm{min}, 175^{\circ} \mathrm{C} \\
100 \mathrm{kPa})\end{array}$ \\
\hline 41 & & & 39.5 & $35.4(R)$ major $^{d}$ & $37.4(S)$ minor & $\begin{array}{l}\mathrm{GC}-\mathrm{MS}\left(\mathrm{G}-\mathrm{TA}, 60^{\circ} \mathrm{C},\right. \\
30 \mathrm{~min}, 3^{\circ} \mathrm{C} / \mathrm{min}, 175^{\circ} \mathrm{C}, \\
100 \mathrm{kPa}) .\end{array}$ \\
\hline 42 & & & 39.0 & $33.9(S)$ major $^{\mathrm{e}}$ & $34.3(R)$ minor & $\begin{array}{l}\mathrm{GC}-\mathrm{MS}\left(\mathrm{G}-\mathrm{TA}, 60^{\circ} \mathrm{C}\right. \\
30 \mathrm{~min}, 3^{\circ} \mathrm{C} / \mathrm{min}, 175^{\circ} \mathrm{C} \\
100 \mathrm{kPa})\end{array}$ \\
\hline 43 & & & 13.0 & $8.9(R)$ minor & $10.5(S)$ major $^{a}$ & $\begin{array}{l}\text { GC (CP-Chirasil-Dex CB, } \\
\left.130^{\circ} \mathrm{C}\right)^{\mathrm{g}}\end{array}$ \\
\hline 44 & & & 21.1 & $11.2(S)$ major $^{f}$ & $13.8(R)$ minor & $\begin{array}{l}\text { HPLC (chiracel OB-H } \\
\text { column, } 254 \mathrm{~nm}, 20^{\circ} \mathrm{C} \text {, } \\
\text { n-hexane: } i-\mathrm{PrOH} \\
\text { 99.5/0.5.) }\end{array}$ \\
\hline 23 & $M$ & & 47.90 & $43.80(S)$ major $^{a}$ & $43.95(R)$ minor & $\begin{array}{l}\text { GC-MS (G-TA, } 60^{\circ} \mathrm{C}, \\
30 \mathrm{~min}, 3^{\circ} \mathrm{C} / \mathrm{min}, 175^{\circ} \mathrm{C} \\
100 \mathrm{kPa}) .\end{array}$ \\
\hline 24 & & & 47.93 & $43.80(S)$ minor & $43.95(R)$ major $^{\mathrm{a}}$ & $\begin{array}{l}\mathrm{GC}-\mathrm{MS}\left(\mathrm{G}-\mathrm{TA}, 60^{\circ} \mathrm{C},\right. \\
30 \mathrm{~min}, 3^{\circ} \mathrm{C} / \mathrm{min}, 175^{\circ} \mathrm{C}, \\
100 \mathrm{kPa}) .\end{array}$ \\
\hline
\end{tabular}

Absolute configurations of products given in paranthesis after retention values. Helium was used as carrier gas for all GC-separations. ${ }^{a}$ Lightfoot, A.; Schnider, P.; Pfaltz, A. Angew. Chem. Int. Ed. 1998, 37, 2897. ${ }^{b}$ Marks T. J. Conticello, V.P. Brard, L. Giardello M. A., Tsuji, Y., Sabat, M. .

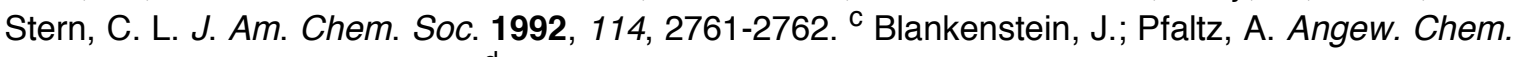
Int. Ed. 2001, 40, 4445-4447. ' Takaya, H., Ohata, T. Ikegami, H., Miyake, T. J. Organometal. Chem. 1995, 502, 169-176. ${ }^{e}$ Ito, Y. hayashi, T. Kawamura, N. Tetrahedron letters, 1988, 29, 59695972. ' McManus, S. P. Colwell, A. R. Duckwall, L. R. Brooks, R. J. Org. Chem. 1981, 46, 30973102. ${ }^{9}$ FID-detector.

\title{
Computational details
}

\author{
pi-bonded olefin \\ Ir_PhThiaz_HtN_Haxu_H2axd_SPhMe2Hd
}

Energy: -2295.17935 hartrees 
The zero point energy (ZPE): $413.645 \mathrm{kcal} / \mathrm{mol}$ final geometry:

angstroms

$\begin{array}{llll}\text { atom } & \mathrm{x} & \mathrm{y} & \mathrm{z}\end{array}$

\begin{tabular}{|c|c|c|c|}
\hline $\mathrm{H} 1$ & 0.1862879469 & 0.0728873390 & 0.0844580352 \\
\hline $\mathrm{C} 2$ & 0.2665792753 & 0.0398536832 & 1.4691808162 \\
\hline H3 & 3.3669169273 & -0.3605639359 & -0.9831877334 \\
\hline $\mathrm{H} 4$ & 3.4318750062 & -0.4631948821 & -0.0308908198 \\
\hline S5 & 0.9699760844 & -4.9357543480 & 0.1920422765 \\
\hline C6 & 1.8499030084 & -3.4388453590 & 0.2790487283 \\
\hline N7 & 1.4023269634 & -2.5196958270 & -0.5637058092 \\
\hline $\mathrm{C} 8$ & 0.3649068477 & -3.0128353602 & -1.3606201027 \\
\hline C9 & -0.2526587121 & -2.2265941102 & -2.5016160393 \\
\hline $\mathrm{C} 10$ & -1.1249347787 & -3.1586842787 & -3.3765296888 \\
\hline $\mathrm{C} 11$ & -2.0084705930 & -4.0980613722 & -2.5485651653 \\
\hline $\mathrm{C} 12$ & -1.1383327635 & -5.0618521898 & -1.7255788703 \\
\hline $\mathrm{C} 13$ & -0.0262681527 & -4.2923637301 & -1.0696185250 \\
\hline H14 & -0.4688394345 & -3.7574629083 & -4.0234649444 \\
\hline H15 & -2.6433976600 & -3.5120932937 & -1.8722257534 \\
\hline H16 & -1.7395188312 & -5.5815217219 & -0.9707813524 \\
\hline H17 & -0.9138446472 & -1.4567079548 & -2.0771511814 \\
\hline H18 & -1.7371314263 & -2.5372160031 & -4.0389053348 \\
\hline H19 & -2.6753449916 & -4.6685683929 & -3.2025314654 \\
\hline $\mathrm{H} 20$ & -0.7170689741 & -5.8407952084 & -2.3760395962 \\
\hline $\mathrm{C} 21$ & 0.8128900636 & -1.5308538209 & -3.3864197870 \\
\hline $\mathrm{H} 22$ & 1.6992080104 & -2.1652176571 & -3.4866422445 \\
\hline $\mathrm{H} 23$ & 0.4171543418 & -1.3725141066 & -4.3941628319 \\
\hline $\mathrm{P} 24$ & 1.3258639698 & 0.0940876413 & -2.6669521544 \\
\hline $\mathrm{C} 25$ & 4.9213380334 & 1.6568592426 & -5.1537346858 \\
\hline $\mathrm{C} 26$ & 4.9060278773 & 0.3400997349 & -4.6868214678 \\
\hline $\mathrm{C} 27$ & 3.8128683317 & -0.1339772107 & -3.9630577202 \\
\hline
\end{tabular}




\begin{tabular}{|c|c|c|c|}
\hline 28 & 2.7133762778 & 0.7018053331 & -3.7061105282 \\
\hline $\mathrm{C} 29$ & 2.7361941592 & 2.0217273598 & -4.1783218595 \\
\hline $\mathrm{C} 30$ & 3.8365387425 & 2.4942478229 & -4.8960820298 \\
\hline H31 & 5.7756663247 & 2.0267605579 & -5.7125344598 \\
\hline H32 & 5.7466432505 & -0.3183668851 & -4.8855302005 \\
\hline H33 & 3.8249235875 & -1.1590309461 & -3.6002143616 \\
\hline H34 & 1.8985452829 & 2.6849328871 & -3.9890752727 \\
\hline H35 & 3.8419222216 & 3.5198937458 & -5.2532491952 \\
\hline Ir36 & 1.8183205818 & -0.2234148866 & -0.3653867058 \\
\hline C37 & -2.2709243418 & 2.8539954114 & -3.6690794701 \\
\hline C38 & -1.7131235185 & 2.8717444453 & -2.3915815408 \\
\hline C39 & -0.6279319105 & 2.0468085003 & -2.0898879267 \\
\hline $\mathrm{C} 40$ & -0.0888958691 & 1.1950657392 & -3.0633800969 \\
\hline C41 & -0.6463159001 & 1.1995505900 & -4.3543458559 \\
\hline $\mathrm{C} 42$ & -1.7329591884 & 2.0192379331 & -4.6514264973 \\
\hline H43 & -3.1184518683 & 3.4916672780 & -3.9026458656 \\
\hline H44 & -2.1200201846 & 3.5285018127 & -1.6280979951 \\
\hline $\mathrm{H} 45$ & -0.1893289076 & 2.0708127938 & -1.0981343081 \\
\hline H46 & -0.2294101244 & 0.5742753525 & -5.1385645833 \\
\hline H47 & -2.1571500154 & 2.0091418816 & -5.6511767677 \\
\hline H48 & 2.0870852456 & 1.3199004444 & -0.2484751250 \\
\hline C49 & 5.2525625270 & -3.3039735247 & 2.8697668522 \\
\hline $\mathrm{C} 50$ & 5.3704477312 & -2.8978511030 & 1.5384829328 \\
\hline C51 & 4.2609993386 & -2.9259409202 & 0.6961083465 \\
\hline C52 & 3.0138703800 & -3.3545557768 & 1.1804812671 \\
\hline C53 & 2.9096173615 & -3.7841924650 & 2.5143636911 \\
\hline C54 & 4.0226443381 & -3.7529663186 & 3.3532225358 \\
\hline H55 & 6.1189338896 & -3.2804235099 & 3.5240438910 \\
\hline H56 & 6.3318310659 & -2.5722325115 & 1.1521067767 \\
\hline H57 & 4.3631669666 & -2.6518675587 & -0.3488679109 \\
\hline H58 & 1.9509260345 & -4.1239517862 & 2.8966223310 \\
\hline
\end{tabular}




\begin{tabular}{|c|c|c|c|}
\hline H59 & 3.9281429814 & -4.0771904961 & 4.3852406258 \\
\hline C60 & 1.6621459299 & -0.2455995675 & 1.8140628238 \\
\hline H61 & 1.8260014884 & -1.2707465367 & 2.1318536368 \\
\hline C63 & -2.7919350277 & -2.8792273430 & 2.3784073384 \\
\hline C64 & -2.9254085092 & -2.0512321183 & 1.2611966914 \\
\hline C65 & -1.9241843590 & -1.1354817913 & 0.9468883912 \\
\hline C66 & -0.7683317605 & -1.0364811855 & 1.7354456228 \\
\hline C67 & -0.6497825355 & -1.8638385771 & 2.8606612591 \\
\hline C68 & -1.6550552944 & -2.7795547893 & 3.1779913057 \\
\hline H69 & -3.5744085114 & -3.5895856794 & 2.6282674358 \\
\hline $\mathrm{H} 70$ & -3.8146644624 & -2.1136600469 & 0.6403722100 \\
\hline H71 & -2.0421048387 & -0.4887152719 & 0.0796641633 \\
\hline H72 & 0.2168864796 & -1.7781826655 & 3.5087750699 \\
\hline H73 & -1.5508878805 & -3.4070448538 & 4.0584755377 \\
\hline C74 & -0.2686614086 & 1.4482644615 & 1.7471378081 \\
\hline $\mathrm{H} 75$ & -1.2292863082 & 1.6137822136 & 1.2539246489 \\
\hline H76 & -0.4318030564 & 1.5421052940 & 2.8264006461 \\
\hline H77 & 0.4331976272 & 2.2218127316 & 1.4335780520 \\
\hline $\mathrm{C} 78$ & 2.5032861676 & 0.7453169739 & 2.5982014534 \\
\hline H79 & 3.5391247796 & 0.3995013097 & 2.6469650661 \\
\hline H80 & 2.5091221987 & 1.7448795152 & 2.1599942266 \\
\hline H81 & 2.1375557169 & 0.8322337122 & 3.6310165355 \\
\hline X82 & 0.9899895363 & -0.1081147491 & 1.6479549237 \\
\hline
\end{tabular}

\section{TS-MI}

Ir_PhThiaz_HtN_Haxu_H2axd_SphMe2Hd_MI1

Energy: -2295.17935 hartrees

The zero point energy (ZPE): $413.645 \mathrm{kcal} / \mathrm{mol}$ final geometry:

angstroms

atom $\quad \mathrm{x} \quad \mathrm{y} \quad \mathrm{z}$

H1 $\quad 0.1862879469 \quad 0.0728873390 \quad 0.0844580352$ 


\begin{tabular}{|c|c|c|c|}
\hline 2 & 0.2665792753 & 0.0398536832 & 1.4691808162 \\
\hline $\mathrm{H} 3$ & 3.3669169273 & -0.3605639359 & -0.9831877334 \\
\hline H4 & 3.4318750062 & -0.4631948821 & -0.0308908198 \\
\hline S5 & 0.9699760844 & -4.9357543480 & 0.1920422765 \\
\hline C6 & 1.8499030084 & -3.4388453590 & 0.2790487283 \\
\hline N7 & 1.4023269634 & -2.5196958270 & -0.5637058092 \\
\hline $\mathrm{C} 8$ & 0.3649068477 & -3.0128353602 & -1.3606201027 \\
\hline C9 & -0.2526587121 & -2.2265941102 & -2.5016160393 \\
\hline $\mathrm{C} 10$ & -1.1249347787 & -3.1586842787 & -3.3765296888 \\
\hline C11 & -2.0084705930 & -4.0980613722 & -2.5485651653 \\
\hline $\mathrm{C} 12$ & -1.1383327635 & -5.0618521898 & -1.7255788703 \\
\hline $\mathrm{C} 13$ & -0.0262681527 & -4.2923637301 & -1.0696185250 \\
\hline H14 & -0.4688394345 & -3.7574629083 & -4.0234649444 \\
\hline H15 & -2.6433976600 & -3.5120932937 & -1.8722257534 \\
\hline H16 & -1.7395188312 & -5.5815217219 & -0.9707813524 \\
\hline $\mathrm{H} 17$ & -0.9138446472 & -1.4567079548 & -2.0771511814 \\
\hline H18 & -1.7371314263 & -2.5372160031 & -4.0389053348 \\
\hline H19 & -2.6753449916 & -4.6685683929 & -3.2025314654 \\
\hline $\mathrm{H} 20$ & -0.7170689741 & -5.8407952084 & -2.3760395962 \\
\hline $\mathrm{C} 21$ & 0.8128900636 & -1.5308538209 & -3.3864197870 \\
\hline $\mathrm{H} 22$ & 1.6992080104 & -2.1652176571 & -3.4866422445 \\
\hline $\mathrm{H} 23$ & 0.4171543418 & -1.3725141066 & -4.3941628319 \\
\hline P24 & 1.3258639698 & 0.0940876413 & -2.6669521544 \\
\hline $\mathrm{C} 25$ & 4.9213380334 & 1.6568592426 & -5.1537346858 \\
\hline $\mathrm{C} 26$ & 4.9060278773 & 0.3400997349 & -4.6868214678 \\
\hline $\mathrm{C} 27$ & 3.8128683317 & -0.1339772107 & -3.9630577202 \\
\hline $\mathrm{C} 28$ & 2.7133762778 & 0.7018053331 & -3.7061105282 \\
\hline $\mathrm{C} 29$ & 2.7361941592 & 2.0217273598 & -4.1783218595 \\
\hline $\mathrm{C} 30$ & 3.8365387425 & 2.4942478229 & -4.8960820298 \\
\hline H31 & 5.7756663247 & 2.0267605579 & -5.7125344598 \\
\hline H32 & 5.7466432505 & -0.3183668851 & -4.8855302005 \\
\hline
\end{tabular}




\begin{tabular}{|c|c|c|c|}
\hline H33 & 3.8249235875 & -1.1590309461 & -3.6002143616 \\
\hline H34 & 1.8985452829 & 2.6849328871 & -3.9890752727 \\
\hline H35 & 3.8419222216 & 3.5198937458 & -5.2532491952 \\
\hline $\operatorname{Ir} 36$ & 1.8183205818 & -0.2234148866 & -0.3653867058 \\
\hline $\mathrm{C} 37$ & -2.2709243418 & 2.8539954114 & -3.6690794701 \\
\hline $\mathrm{C} 38$ & -1.7131235185 & 2.8717444453 & -2.3915815408 \\
\hline C39 & -0.6279319105 & 2.0468085003 & -2.0898879267 \\
\hline $\mathrm{C} 40$ & -0.0888958691 & 1.1950657392 & -3.0633800969 \\
\hline $\mathrm{C} 41$ & -0.6463159001 & 1.1995505900 & -4.3543458559 \\
\hline $\mathrm{C} 42$ & -1.7329591884 & 2.0192379331 & -4.6514264973 \\
\hline $\mathrm{H} 43$ & -3.1184518683 & 3.4916672780 & -3.9026458656 \\
\hline H44 & -2.1200201846 & 3.5285018127 & -1.6280979951 \\
\hline H45 & -0.1893289076 & 2.0708127938 & -1.0981343081 \\
\hline H46 & -0.2294101244 & 0.5742753525 & -5.1385645833 \\
\hline $\mathrm{H} 47$ & -2.1571500154 & 2.0091418816 & -5.6511767677 \\
\hline H48 & 2.0870852456 & 1.3199004444 & -0.2484751250 \\
\hline C49 & 5.2525625270 & -3.3039735247 & 2.8697668522 \\
\hline $\mathrm{C} 50$ & 5.3704477312 & -2.8978511030 & 1.5384829328 \\
\hline $\mathrm{C} 51$ & 4.2609993386 & -2.9259409202 & 0.6961083465 \\
\hline C52 & 3.0138703800 & -3.3545557768 & 1.1804812671 \\
\hline C53 & 2.9096173615 & -3.7841924650 & 2.5143636911 \\
\hline $\mathrm{C} 54$ & 4.0226443381 & -3.7529663186 & 3.3532225358 \\
\hline H55 & 6.1189338896 & -3.2804235099 & 3.5240438910 \\
\hline H56 & 6.3318310659 & -2.5722325115 & 1.1521067767 \\
\hline H57 & 4.3631669666 & -2.6518675587 & -0.3488679109 \\
\hline H58 & 1.9509260345 & -4.1239517862 & 2.8966223310 \\
\hline H59 & 3.9281429814 & -4.0771904961 & 4.3852406258 \\
\hline C60 & 1.6621459299 & -0.2455995675 & 1.8140628238 \\
\hline H61 & 1.8260014884 & -1.2707465367 & 2.1318536368 \\
\hline C63 & -2.7919350277 & -2.8792273430 & 2.3784073384 \\
\hline C64 & -2.9254085092 & -2.0512321183 & 1.2611966914 \\
\hline
\end{tabular}




\begin{tabular}{|c|c|c|c|}
\hline C65 & -1.9241843590 & -1.1354817913 & 0.9468883912 \\
\hline C66 & -0.7683317605 & -1.0364811855 & 1.7354456228 \\
\hline C67 & -0.6497825355 & -1.8638385771 & 2.8606612591 \\
\hline C68 & -1.6550552944 & -2.7795547893 & 3.1779913057 \\
\hline H69 & -3.5744085114 & -3.5895856794 & 2.6282674358 \\
\hline $\mathrm{H} 70$ & -3.8146644624 & -2.1136600469 & 0.6403722100 \\
\hline H71 & -2.0421048387 & -0.4887152719 & 0.0796641633 \\
\hline $\mathrm{H} 72$ & 0.2168864796 & -1.7781826655 & 3.5087750699 \\
\hline H73 & -1.5508878805 & -3.4070448538 & 4.0584755377 \\
\hline C74 & -0.2686614086 & 1.4482644615 & 1.7471378081 \\
\hline $\mathrm{H} 75$ & -1.2292863082 & 1.6137822136 & 1.2539246489 \\
\hline H76 & -0.4318030564 & 1.5421052940 & 2.8264006461 \\
\hline $\mathrm{H} 77$ & 0.4331976272 & 2.2218127316 & 1.4335780520 \\
\hline $\mathrm{C} 78$ & 2.5032861676 & 0.7453169739 & 2.5982014534 \\
\hline H79 & 3.5391247796 & 0.3995013097 & 2.6469650661 \\
\hline H80 & 2.5091221987 & 1.7448795152 & 2.1599942266 \\
\hline H81 & 2.1375557169 & 0.8322337122 & 3.6310165355 \\
\hline X82 & 0.9899895363 & -0.1081147491 & 1.6479549237 \\
\hline
\end{tabular}

\section{MI-prod}

Ir_PhThiaz_HtN_Haxu_H2axd_SphMe2Hd_prod

energy: -2295.18858 hartrees

The zero point energy (ZPE): $416.344 \mathrm{kcal} / \mathrm{mol}$

final geometry:

angstroms

$\begin{array}{lccc}\text { atom } & \mathrm{x} & \mathrm{y} & \mathrm{z} \\ \mathrm{H} 1 & 0.0612358683 & 0.0876477024 & -0.0388462697 \\ \text { C2 } & 0.0352018256 & 0.1169287153 & 1.1495128651 \\ \text { H3 } & 3.2640285129 & -0.3766809815 & -1.2209523759 \\ \text { H4 } & 3.1502367400 & -0.4150361204 & 0.4962334600 \\ \text { S5 } & 1.4960649608 & -4.9903488628 & 0.1011875983 \\ \text { C6 } & 2.1161965389 & -3.3756274689 & 0.2626294167\end{array}$




\begin{tabular}{|c|c|c|c|}
\hline N7 & 1.6027863007 & -2.5268122374 & -0.6172329584 \\
\hline $\mathrm{C} 8$ & 0.7199474175 & -3.1663274186 & -1.4950549982 \\
\hline C9 & 0.0205080733 & -2.4658196628 & -2.6464446128 \\
\hline $\mathrm{C} 10$ & -0.6400531756 & -3.5095236647 & -3.5803968113 \\
\hline $\mathrm{C} 11$ & -1.3871512173 & -4.6104307286 & -2.8217230759 \\
\hline $\mathrm{C} 12$ & -0.4040853570 & -5.4296416218 & -1.9721361313 \\
\hline $\mathrm{C} 13$ & 0.5178801310 & -4.4967746468 & -1.2377641313 \\
\hline $\mathrm{H} 14$ & 0.1380481375 & -3.9657618487 & -4.2078470521 \\
\hline H15 & -2.1490016500 & -4.1633244272 & -2.1714143421 \\
\hline H16 & -0.9408311746 & -6.0710885550 & -1.2641464314 \\
\hline $\mathrm{H} 17$ & -0.7829642217 & -1.8486457868 & -2.2205238309 \\
\hline $\mathrm{H} 18$ & -1.3213939257 & -2.9854745364 & -4.2603102627 \\
\hline H19 & -1.9090465468 & -5.2682104198 & -3.5237777622 \\
\hline $\mathrm{H} 20$ & 0.1831365844 & -6.1005473650 & -2.6142484206 \\
\hline $\mathrm{C} 21$ & 0.9517137646 & -1.5542041123 & -3.4893354640 \\
\hline $\mathrm{H} 22$ & 1.9478771788 & -1.9987882142 & -3.5868714919 \\
\hline $\mathrm{H} 23$ & 0.5539027825 & -1.4495563816 & -4.5039520736 \\
\hline $\mathrm{P} 24$ & 1.1875990691 & 0.1053362186 & -2.7265980011 \\
\hline $\mathrm{C} 25$ & 3.9736842608 & 2.5164545907 & -5.5265738875 \\
\hline $\mathrm{C} 26$ & 4.3119985932 & 2.3638658117 & -4.1827672121 \\
\hline $\mathrm{C} 27$ & 3.4882155421 & 1.6260879987 & -3.3308023044 \\
\hline $\mathrm{C} 28$ & 2.3168633121 & 1.0351679666 & -3.8237811612 \\
\hline $\mathrm{C} 29$ & 1.9758229966 & 1.2025495463 & -5.1770988116 \\
\hline $\mathrm{C} 30$ & 2.8044237131 & 1.9343798728 & -6.0233772971 \\
\hline H31 & 4.6143412498 & 3.0938083025 & -6.1865763297 \\
\hline $\mathrm{H} 32$ & 5.2148744329 & 2.8230442885 & -3.7913015205 \\
\hline H33 & 3.7478726921 & 1.5216079434 & -2.2831019308 \\
\hline H34 & 1.0553844051 & 0.7796139941 & -5.5706112996 \\
\hline H35 & 2.5338265199 & 2.0586034963 & -7.0677096562 \\
\hline Ir36 & 1.9007815482 & -0.2238994247 & -0.4610740285 \\
\hline $\mathrm{C} 37$ & -2.8024014221 & 2.4421263232 & -3.1738966628 \\
\hline
\end{tabular}




\begin{tabular}{|c|c|c|c|}
\hline $\mathrm{C} 38$ & -1.7631032605 & 2.9341452385 & -2.3807926851 \\
\hline C39 & -0.5828024588 & 2.2064687394 & -2.2423111950 \\
\hline $\mathrm{C} 40$ & -0.4229050037 & 0.9767462710 & -2.9035719072 \\
\hline $\mathrm{C} 41$ & -1.4680313324 & 0.4955351827 & -3.7066618274 \\
\hline $\mathrm{C} 42$ & -2.6523746662 & 1.2241675952 & -3.8352857122 \\
\hline H43 & -3.7237874105 & 3.0076287349 & -3.2770613423 \\
\hline H44 & -1.8719438948 & 3.8864033199 & -1.8697973135 \\
\hline $\mathrm{H} 45$ & 0.2204623024 & 2.5966289017 & -1.6234460613 \\
\hline H46 & -1.3732113291 & -0.4437814835 & -4.2411783882 \\
\hline H47 & -3.4550265414 & 0.8380129086 & -4.4569503334 \\
\hline $\mathrm{H} 48$ & 2.2012524447 & 1.3159910028 & -0.3446645574 \\
\hline C49 & 5.1797237310 & -2.8099907206 & 3.1952213267 \\
\hline $\mathrm{C} 50$ & 5.4044732951 & -2.4285211297 & 1.8702786904 \\
\hline C51 & 4.4043966836 & -2.5912107928 & 0.9134000161 \\
\hline C52 & 3.1609821779 & -3.1351265130 & 1.2774047149 \\
\hline C53 & 2.9506557599 & -3.5380503784 & 2.6068801162 \\
\hline C54 & 3.9537610881 & -3.3684080784 & 3.5604220748 \\
\hline H55 & 5.9612191568 & -2.6803198889 & 3.9381455491 \\
\hline H56 & 6.3644971530 & -2.0128337574 & 1.5786605785 \\
\hline H57 & 4.5887738123 & -2.3205246666 & -0.1206995353 \\
\hline H58 & 1.9944309160 & -3.9663988024 & 2.8949233896 \\
\hline H59 & 3.7767989660 & -3.6717870780 & 4.5879656415 \\
\hline C60 & 1.4242960827 & -0.1852572552 & 1.6594929967 \\
\hline H61 & 1.5163255816 & -1.2070404279 & 2.0176727422 \\
\hline C63 & -3.0625010211 & -2.7576419064 & 2.1091831651 \\
\hline C64 & -3.0652422289 & -2.1070570144 & 0.8737943427 \\
\hline C65 & -2.0517341901 & -1.2034836132 & 0.5577295221 \\
\hline C66 & -1.0161423344 & -0.9411349980 & 1.4649397612 \\
\hline C67 & -1.0292789692 & -1.5895466960 & 2.7066956416 \\
\hline C68 & -2.0453190086 & -2.4925063592 & 3.0253567263 \\
\hline H69 & -3.8527754636 & -3.4595254373 & 2.3590234728 \\
\hline
\end{tabular}




$\begin{array}{llll}\text { H70 } & -3.8617777570 & -2.2975955324 & 0.1598479586 \\ \text { H71 } & -2.0733555292 & -0.6860335999 & -0.3996678363 \\ \text { H72 } & -0.2557351555 & -1.3792824784 & 3.4401266165 \\ \text { H73 } & -2.0435621622 & -2.9834150253 & 3.9944809958 \\ \text { C74 } & -0.4850393623 & 1.5431524501 & 1.4221066958 \\ \text { H75 } & -1.4256222477 & 1.7136639773 & 0.8931704863 \\ \text { H76 } & -0.6750795614 & 1.6628876206 & 2.4930736009 \\ \text { H77 } & 0.2346357541 & 2.3014226345 & 1.1074509815 \\ \text { C78 } & 2.1214963311 & 0.7969281145 & 2.5803734576 \\ \text { H79 } & 3.1268592206 & 0.4417325269 & 2.8201383579 \\ \text { H80 } & 2.2112010325 & 1.7955034559 & 2.1494830014 \\ \text { H81 } & 1.5674175568 & 0.8854771074 & 3.5253905151 \\ \text { X82 } & 0.7750468344 & -0.0440184493 & 1.4211332055\end{array}$

TS-RE

Ir_PhThiaz_HtN_Haxu_H2axd_SphMe2Hd_RE1

energy: -2295.18628 hartrees

The zero point energy (ZPE): $415.919 \mathrm{kcal} / \mathrm{mol}$

final geometry:

angstroms

$\begin{array}{lccc}\text { atom } & \mathrm{x} & \mathrm{z} & \\ \text { H1 } & 0.3694585527 & 0.0614719787 & -0.3116292769 \\ \text { C2 } & -0.0534413201 & -0.0451885362 & 1.3448127174 \\ \text { Ir3 } & 1.8788421884 & 0.0097362625 & 0.2467233801 \\ \text { H4 } & 2.0019048736 & -0.0322794803 & -1.3107903972 \\ \text { H5 } & 1.9973203975 & -0.0053271248 & 2.2021986644 \\ \text { C6 } & 0.8883879831 & -0.1110517869 & 2.5500770789 \\ \text { S7 } & 1.2910175033 & 4.7920709056 & 0.2886151316 \\ \text { C8 } & 0.9796196781 & 3.1135648495 & -0.0326588603 \\ \text { N9 } & 2.0250163509 & 2.3315039361 & 0.2000675229 \\ \text { C10 } & 3.1434691009 & 3.0742395663 & 0.5998460092 \\ \text { C11 } & 4.5073346639 & 2.4656052757 & 0.8741181548\end{array}$




\begin{tabular}{|c|c|c|c|}
\hline $\mathrm{C} 12$ & 5.5754036021 & 3.5820796683 & 0.9844602803 \\
\hline $\mathrm{C} 13$ & 5.1160777923 & 4.7713216754 & 1.8334856999 \\
\hline $\mathrm{C} 14$ & 3.9100634624 & 5.4601669538 & 1.1770485111 \\
\hline $\mathrm{C} 15$ & 2.9220426429 & 4.4201432451 & 0.7280740397 \\
\hline H16 & 5.8264681937 & 3.9328879813 & -0.0259950945 \\
\hline H17 & 4.8401584618 & 4.4266302997 & 2.8377244999 \\
\hline H18 & 3.4357028397 & 6.1619465153 & 1.8723010499 \\
\hline H19 & 4.4603026270 & 1.9584774095 & 1.8490377395 \\
\hline $\mathrm{H} 20$ & 6.4905566538 & 3.1467182362 & 1.4010676337 \\
\hline $\mathrm{H} 21$ & 5.9324917850 & 5.4899745664 & 1.9549442047 \\
\hline $\mathrm{H} 22$ & 4.2380058725 & 6.0546885564 & 0.3132459528 \\
\hline $\mathrm{C} 23$ & 4.9579099340 & 1.4376396213 & -0.1960778858 \\
\hline $\mathrm{H} 24$ & 4.6412686933 & 1.7535127912 & -1.1960041152 \\
\hline $\mathrm{H} 25$ & 6.0505955528 & 1.3736793105 & -0.2186980526 \\
\hline P26 & 4.2262435862 & -0.2279819115 & 0.0834843455 \\
\hline $\mathrm{C} 27$ & 5.8835974544 & -2.9935937655 & -3.2367674722 \\
\hline $\mathrm{C} 28$ & 4.5060745391 & -2.8956338579 & -3.0451126595 \\
\hline $\mathrm{C} 29$ & 3.9924224236 & -2.0535552318 & -2.0571067904 \\
\hline $\mathrm{C} 30$ & 4.8605430834 & -1.3031851716 & -1.2522807109 \\
\hline C31 & 6.2484086643 & -1.4153421252 & -1.4437440072 \\
\hline $\mathrm{C} 32$ & 6.7549051410 & -2.2517037634 & -2.4348466339 \\
\hline H33 & 6.2805587906 & -3.6512194173 & -4.0044616787 \\
\hline H34 & 3.8263217461 & -3.4777655625 & -3.6603210558 \\
\hline H35 & 2.9210256257 & -1.9887064378 & -1.9031274159 \\
\hline H36 & 6.9391665660 & -0.8656156717 & -0.8102608395 \\
\hline H37 & 7.8285664001 & -2.3318840894 & -2.5762801821 \\
\hline C38 & 6.1989578326 & -1.9799975176 & 3.9079766087 \\
\hline C39 & 5.0728857255 & -2.5793817092 & 3.3397551969 \\
\hline $\mathrm{C} 40$ & 4.4861267824 & -2.0299668566 & 2.2014836485 \\
\hline $\mathrm{C} 41$ & 5.0244136482 & -0.8748836819 & 1.6104554214 \\
\hline $\mathrm{C} 42$ & 6.1622423393 & -0.2857590090 & 2.1820808884 \\
\hline
\end{tabular}




\begin{tabular}{|c|c|c|c|}
\hline $\mathrm{C} 43$ & 6.7420380239 & -0.8349444981 & 3.3269598181 \\
\hline H44 & 6.6519578665 & -2.4053751223 & 4.7985935873 \\
\hline $\mathrm{H} 45$ & 4.6506812135 & -3.4758660932 & 3.7844505910 \\
\hline H46 & 3.6088632837 & -2.5007905736 & 1.7671975015 \\
\hline H47 & 6.6099793349 & 0.6007294892 & 1.7452595604 \\
\hline $\mathrm{H} 48$ & 7.6201302136 & -0.3659607171 & 3.7615057429 \\
\hline H49 & 1.7639717733 & -1.5596250101 & 0.1980478343 \\
\hline C50 & -2.8657491087 & 2.1587189354 & -1.6490660638 \\
\hline C51 & -1.7088629776 & 1.7196802743 & -2.2971872560 \\
\hline C52 & -0.4527029096 & 2.0123929831 & -1.7687297342 \\
\hline C53 & -0.3427761735 & 2.7472816895 & -0.5759520769 \\
\hline C54 & -1.5108109181 & 3.2042012740 & 0.0586674512 \\
\hline C55 & -2.7639424904 & 2.9046105247 & -0.4736520384 \\
\hline H56 & -3.8424622639 & 1.9268098507 & -2.0636239039 \\
\hline H57 & -1.7840411314 & 1.1564984337 & -3.2227561803 \\
\hline H58 & 0.4454602369 & 1.6909180884 & -2.2847628527 \\
\hline H59 & -1.4353309349 & 3.7730148315 & 0.9813224803 \\
\hline H60 & -3.6602357388 & 3.2517457693 & 0.0316694028 \\
\hline H61 & -0.5329083476 & 0.9282726896 & 1.2820682103 \\
\hline C62 & 0.4006852056 & 3.1234079123 & 5.3917877584 \\
\hline C63 & 1.6789866797 & 2.7291047076 & 4.9954359665 \\
\hline C64 & 1.8376259167 & 1.7063205549 & 4.0601421279 \\
\hline C65 & 0.7235192105 & 1.0664164834 & 3.5049522213 \\
\hline C66 & -0.5567587527 & 1.4658005155 & 3.9136567376 \\
\hline C67 & -0.7166027466 & 2.4868045547 & 4.8506710500 \\
\hline H68 & 0.2754626560 & 3.9176205229 & 6.1218293306 \\
\hline H69 & 2.5536537214 & 3.2149761396 & 5.4185498821 \\
\hline $\mathrm{H} 70$ & 2.8385344373 & 1.3955414374 & 3.7670445870 \\
\hline H71 & -1.4369132411 & 0.9696772366 & 3.5123774709 \\
\hline $\mathrm{H} 72$ & -1.7151935267 & 2.7811635525 & 5.1609395353 \\
\hline C73 & 0.8980118969 & -1.4554553851 & 3.306413740 \\
\hline
\end{tabular}




\begin{tabular}{lrrr} 
H74 & 1.6805581525 & -1.4482857165 & 4.0692266301 \\
H75 & -0.0600964854 & -1.6083934561 & 3.8117057100 \\
H76 & 1.0769948294 & -2.2960383531 & 2.6327081620 \\
C77 & -1.0524567417 & -1.1705118542 & 1.1324164088 \\
H78 & -1.6525631751 & -0.9863691328 & 0.2373929204 \\
H79 & -0.5726452523 & -2.1444314837 & 1.0240378596 \\
H80 & -1.7399564392 & -1.2236022034 & 1.9863681175 \\
X81 & 0.0000000000 & 0.0000000000 & 1.4295230000 \\
& \multicolumn{2}{c}{ RE-prod } \\
Ir_PhThiaz_HtN_Haxu_H2axd_SphMe2Hd_REprod
\end{tabular}

energy: -2295.20503 hartrees

The zero point energy (ZPE): $418.259 \mathrm{kcal} / \mathrm{mol}$ final geometry:

angstroms

$\begin{array}{lccc}\text { atom } & \mathrm{x} & \mathrm{z} & \\ \text { H1 } & -0.1854981292 & -0.2913375252 & 0.9879927756 \\ \text { C2 } & -0.3071852019 & 0.1480567697 & 2.0154760405 \\ \text { Ir3 } & 1.7352161270 & 0.1418315722 & -0.0056501304 \\ \text { H4 } & 1.4358231857 & 0.0037448825 & -1.5129466863 \\ \text { H5 } & 1.8578548515 & 0.0665384936 & 2.2309158963 \\ \text { C6 } & 0.9574673144 & -0.1575499549 & 2.8667630693 \\ \text { S7 } & 1.0072499606 & 4.8538526890 & -0.2975049178 \\ \text { C8 } & 0.6773703636 & 3.1477966480 & -0.3084836467 \\ \text { N9 } & 1.7744132026 & 2.4045054481 & -0.2502076527 \\ \text { C10 } & 2.9332131124 & 3.1863469869 & -0.2425634089 \\ \text { C11 } & 4.3332390138 & 2.6019565420 & -0.2206671403 \\ \text { C12 } & 5.3690871353 & 3.7087490565 & -0.5366493495 \\ \text { C13 } & 5.1019821958 & 5.0116752892 & 0.2257837465 \\ \text { C14 } & 3.7538685619 & 5.6182415067 & -0.1965265038 \\ \text { C15 } & 2.7101776689 & 4.5376022132 & -0.2387178246 \\ \text { H16 } & 5.3549474751 & 3.9094604456 & -1.6167519769\end{array}$




\begin{tabular}{|c|c|c|c|}
\hline H17 & 5.0944451263 & 4.8151822898 & 1.3053342810 \\
\hline H18 & 3.4526452862 & 6.4150997271 & 0.4927793686 \\
\hline H19 & 4.5285437862 & 2.2429216936 & 0.7995504802 \\
\hline $\mathrm{H} 20$ & 6.3697172860 & 3.3289576902 & -0.3014051851 \\
\hline $\mathrm{H} 21$ & 5.9047978200 & 5.7313288420 & 0.0383596430 \\
\hline $\mathrm{H} 22$ & 3.8414895542 & 6.0838545254 & -1.1877954864 \\
\hline $\mathrm{C} 23$ & 4.5345449471 & 1.4209192528 & -1.2042140542 \\
\hline $\mathrm{H} 24$ & 4.0420008714 & 1.6222508771 & -2.1615121987 \\
\hline $\mathrm{H} 25$ & 5.6007751031 & 1.2925184109 & -1.4211292808 \\
\hline P26 & 3.8476845790 & -0.1751844776 & -0.5911668560 \\
\hline $\mathrm{C} 27$ & 4.7801301082 & -3.3133674999 & -3.8674771171 \\
\hline $\mathrm{C} 28$ & 3.4565879330 & -3.0878506122 & -3.4898362853 \\
\hline $\mathrm{C} 29$ & 3.1553375013 & -2.1430308637 & -2.5070188324 \\
\hline C30 & 4.1833674861 & -1.4135304735 & -1.8937234662 \\
\hline C31 & 5.5172137281 & -1.6542635570 & -2.2694925037 \\
\hline C32 & 5.8101525489 & -2.5945793788 & -3.2545972979 \\
\hline H33 & 5.0114735780 & -4.0511699281 & -4.6297423195 \\
\hline H34 & 2.6528206436 & -3.6513578967 & -3.9541507076 \\
\hline H35 & 2.1261937702 & -1.9840260923 & -2.2052710190 \\
\hline H36 & 6.3321211492 & -1.1240119538 & -1.7837636804 \\
\hline H37 & 6.8433743592 & -2.7728951910 & -3.5369976239 \\
\hline C38 & 6.4146959709 & -1.5412814831 & 3.0233103903 \\
\hline C39 & 5.2315061928 & -2.2046028735 & 2.6919583257 \\
\hline $\mathrm{C} 40$ & 4.4675753644 & -1.7764131227 & 1.6085077992 \\
\hline $\mathrm{C} 41$ & 4.8814884320 & -0.6755719249 & 0.8381047595 \\
\hline $\mathrm{C} 42$ & 6.0790851539 & -0.0222562820 & 1.1716306593 \\
\hline $\mathrm{C} 43$ & 6.8379225931 & -0.4539245527 & 2.2600486098 \\
\hline H44 & 7.0058797931 & -1.8729246158 & 3.8716356060 \\
\hline $\mathrm{H} 45$ & 4.9029911996 & -3.0579552523 & 3.2778675897 \\
\hline $\mathrm{H} 46$ & 3.5482173241 & -2.2967842864 & 1.3588229414 \\
\hline $\mathrm{H} 47$ & 6.4360577612 & 0.8210008310 & 0.5899057289 \\
\hline
\end{tabular}




\begin{tabular}{|c|c|c|c|}
\hline $\mathrm{H} 48$ & 7.7606361250 & 0.0619014558 & 2.5088768454 \\
\hline H49 & 1.6299319247 & -1.4334576044 & 0.1105978892 \\
\hline $\mathrm{C} 50$ & -3.3785074937 & 1.8581683972 & -0.7307035129 \\
\hline $\mathrm{C} 51$ & -2.3796345685 & 1.2885248222 & -1.5225929627 \\
\hline $\mathrm{C} 52$ & -1.0544063927 & 1.6984836187 & -1.3836518713 \\
\hline C53 & -0.7129699670 & 2.6790955741 & -0.4356208226 \\
\hline C54 & -1.7274067959 & 3.2592981145 & 0.3464076956 \\
\hline C55 & -3.0499397761 & 2.8468102351 & 0.1998782430 \\
\hline H56 & -4.4107356990 & 1.5402180780 & -0.8447431026 \\
\hline H57 & -2.6342361514 & 0.5359670072 & -2.2630394789 \\
\hline H58 & -0.2879898588 & 1.2867935247 & -2.0310632350 \\
\hline H59 & -1.4760238232 & 4.0164216881 & 1.0838125201 \\
\hline H60 & -3.8243185148 & 3.2951223071 & 0.8150231880 \\
\hline H61 & -0.3979571694 & 1.2296769957 & 1.9015220919 \\
\hline C62 & 1.1757332809 & 2.7036740490 & 6.1261867948 \\
\hline C63 & 1.7323315919 & 2.9986124972 & 4.8808906935 \\
\hline C64 & 1.6739324364 & 2.0611978012 & 3.8507586735 \\
\hline C65 & 1.0622186056 & 0.8138285198 & 4.0389940806 \\
\hline C66 & 0.5146064364 & 0.5267054415 & 5.2969879755 \\
\hline C67 & 0.5689260645 & 1.4651977008 & 6.3298047216 \\
\hline H68 & 1.2186466782 & 3.4307808328 & 6.9318103423 \\
\hline H69 & 2.2158107695 & 3.9573179679 & 4.7141269821 \\
\hline $\mathrm{H} 70$ & 2.1141974999 & 2.2980655569 & 2.8833048876 \\
\hline H71 & 0.0414029486 & -0.4322550332 & 5.4837532155 \\
\hline $\mathrm{H} 72$ & 0.1357257303 & 1.2238189734 & 7.2963975898 \\
\hline $\mathrm{C} 73$ & 1.0815636824 & -1.6363502500 & 3.2600526833 \\
\hline $\mathrm{H} 74$ & 2.0310832123 & -1.8199983602 & 3.7701119180 \\
\hline $\mathrm{H} 75$ & 0.2784669328 & -1.9449257415 & 3.9342461139 \\
\hline H76 & 1.0321117006 & -2.2768892742 & 2.3746848884 \\
\hline C77 & -1.6307615371 & -0.4076123507 & 2.5557632668 \\
\hline H78 & -2.4635812666 & -0.0713481798 & 1.9325447777 \\
\hline
\end{tabular}




$\begin{array}{llll}\text { H79 } & -1.6431888966 & -1.5009642459 & 2.5745649066 \\ \text { H80 } & -1.8062019091 & -0.0440541760 & 3.5735432956 \\ \text { X81 } & 0.3371639990 & -0.0746469976 & 2.3447020003\end{array}$

TS A

energy: $\quad-2483.34206677414$ hartrees

The zero point energy (ZPE): $\quad 423.253 \mathrm{kcal} / \mathrm{mol}$

final geometry:

angstroms

$\begin{array}{lccc}\text { atom } & \mathrm{x} & \mathrm{y} & \mathrm{z} \\ \text { H1 } & 0.0815000470 & 0.0531283620 & 0.0081443968 \\ \text { C2 } & 0.0593843070 & 0.1312906625 & 1.3541139029 \\ \text { Ir3 } & 1.7827022056 & -0.0433501132 & -0.3303538392 \\ \text { H4 } & 1.8658084918 & 1.5204979848 & -0.2766520866 \\ \text { H5 } & 3.3949779039 & -0.0737552843 & 0.0847682025 \\ \text { H6 } & 3.3683013756 & -0.0385454874 & -0.8638388900 \\ \text { C7 } & 1.4578423306 & 0.0916150626 & 1.8187543459 \\ \text { S8 } & 1.3975134788 & -4.8147626455 & 0.3643451120 \\ \text { C9 } & 2.0765278904 & -3.2180659512 & 0.4748815771 \\ \text { N10 } & 1.6325305853 & -2.3940381490 & -0.4621463696 \\ \text { C11 } & 0.7643397824 & -3.0411487463 & -1.3486070319 \\ \text { C12 } & 0.1870036463 & -2.3808850534 & -2.5883351852 \\ \text { C13 } & -0.4363355928 & -3.4495688615 & -3.5191414529 \\ \text { C14 } & -1.2867049635 & -4.4753962424 & -2.7624501676 \\ \text { C15 } & -0.4141597960 & -5.2814674623 & -1.7869172774 \\ \text { C16 } & 0.4987256804 & -4.3490043831 & -1.0408770375 \\ \text { H17 } & 0.3708422061 & -3.9710912851 & -4.0521743163 \\ \text { H18 } & -2.0791770274 & -3.9609295237 & -2.2047945032 \\ \text { H19 } & -1.0365373178 & -5.8488096257 & -1.0853188054 \\ \text { H20 } & -0.6211027429 & -1.7012017521 & -2.2784390321\end{array}$




\begin{tabular}{|c|c|c|c|}
\hline $\mathrm{H} 21$ & -1.0363871964 & -2.9375319010 & -4.2792173082 \\
\hline $\mathrm{H} 22$ & -1.7794485318 & -5.1542292438 & -3.4655853047 \\
\hline $\mathrm{H} 23$ & 0.1838242262 & -6.0212294927 & -2.3370420829 \\
\hline $\mathrm{C} 24$ & 1.2392976062 & -1.5645512570 & -3.3778303950 \\
\hline $\mathrm{H} 25$ & 2.2087802942 & -2.0723070134 & -3.3601279350 \\
\hline $\mathrm{H} 26$ & 0.9418187111 & -1.4817759563 & -4.4277229902 \\
\hline P27 & 1.4566715454 & 0.1278141224 & -2.6698228420 \\
\hline $\mathrm{C} 28$ & 5.0638360692 & 2.0667355143 & -4.8423133204 \\
\hline $\mathrm{C} 29$ & 5.1648422370 & 0.7726247033 & -4.3257748460 \\
\hline C30 & 4.0694613076 & 0.1844271374 & -3.6973710705 \\
\hline C31 & 2.8548110875 & 0.8823043475 & -3.5867904234 \\
\hline C32 & 2.7605420663 & 2.1796077279 & -4.1086154627 \\
\hline C33 & 3.8633562414 & 2.7669810478 & -4.7314116341 \\
\hline H34 & 5.9198276043 & 2.5268471389 & -5.3268245470 \\
\hline H35 & 6.0972418805 & 0.2222388671 & -4.4114048848 \\
\hline H36 & 4.1698450871 & -0.8202550334 & -3.2933679109 \\
\hline H37 & 1.8319121974 & 2.7354371727 & -4.0293174287 \\
\hline H38 & 3.7805494827 & 3.7745124968 & -5.1282773701 \\
\hline C39 & -2.3354156971 & 2.4501620592 & -4.0103976105 \\
\hline $\mathrm{C} 40$ & -1.8478899521 & 2.5934401841 & -2.7113642969 \\
\hline $\mathrm{C} 41$ & -0.7086739133 & 1.8942801284 & -2.3080270639 \\
\hline $\mathrm{C} 42$ & -0.0416867525 & 1.0432227955 & -3.2009908064 \\
\hline C43 & -0.5296320827 & 0.9197331712 & -4.5141747681 \\
\hline $\mathrm{C} 44$ & -1.6706892020 & 1.6141566167 & -4.9115070788 \\
\hline $\mathrm{H} 45$ & -3.2240063350 & 2.9903014079 & -4.3227632119 \\
\hline H46 & -2.3510064630 & 3.2512296734 & -2.0084284503 \\
\hline $\mathrm{H} 47$ & -0.3287792726 & 2.0206149252 & -1.2999293998 \\
\hline H48 & -0.0183990548 & 0.2926368306 & -5.2388552568 \\
\hline H49 & -2.0382339382 & 1.5053021422 & -5.9276987804 \\
\hline C50 & 5.1190397818 & -2.6115129970 & 3.4181743451 \\
\hline Cग1 & 5.3511435276 & -2.2521967807 & 2.0884956221 \\
\hline
\end{tabular}




\begin{tabular}{|c|c|c|c|}
\hline C52 & 4.3543877264 & -2.4280583947 & 1.1313409994 \\
\hline C53 & 3.1073023628 & -2.9611832919 & 1.4972353991 \\
\hline C54 & 2.8886134682 & -3.3390629795 & 2.8326683698 \\
\hline C55 & 3.8890471502 & -3.1582776900 & 3.7867117010 \\
\hline H56 & 5.8985224230 & -2.4754460005 & 4.1622397534 \\
\hline H57 & 6.3158695381 & -1.8492011082 & 1.7941975804 \\
\hline H58 & 4.5507835748 & -2.1931392285 & 0.0899510956 \\
\hline H59 & 1.9281936554 & -3.7553059544 & 3.1239325977 \\
\hline H60 & 3.7067736198 & -3.4440097643 & 4.8185187732 \\
\hline H61 & 1.7619077212 & -0.8326091070 & 2.2963029337 \\
\hline C62 & -0.7501152797 & 1.4309162177 & 1.4707197369 \\
\hline H63 & -1.6731607833 & 1.3558376152 & 0.8906890949 \\
\hline H64 & -1.0279257797 & 1.5570847759 & 2.5227637142 \\
\hline H65 & -0.1686064235 & 2.2990241398 & 1.1691658097 \\
\hline C66 & 2.1036430526 & 1.2879032522 & 2.4493672400 \\
\hline O67 & 3.0441425167 & 0.8831893364 & 3.3302634412 \\
\hline O68 & 1.8550884683 & 2.4581201294 & 2.2347882801 \\
\hline C69 & 3.7526429617 & 1.9404904920 & 4.0065039427 \\
\hline $\mathrm{H} 70$ & 4.4608945087 & 1.4412257281 & 4.6669914609 \\
\hline H71 & 4.2764210431 & 2.5740129891 & 3.2865244554 \\
\hline $\mathrm{H} 72$ & 3.0594756015 & 2.5579522299 & 4.5827391438 \\
\hline C73 & -2.5086255695 & -3.1769108956 & 2.4246740554 \\
\hline C74 & -2.6329622814 & -2.5765365253 & 1.1701025403 \\
\hline $\mathrm{C} 75$ & -1.7806868075 & -1.5363897295 & 0.8048772707 \\
\hline C76 & -0.7834570120 & -1.0874872280 & 1.6805877795 \\
\hline C77 & -0.6712785795 & -1.6894899328 & 2.9413078279 \\
\hline $\mathrm{C} 78$ & -1.5297047796 & -2.7269696422 & 3.3095321296 \\
\hline H79 & -3.1766671841 & -3.9830133967 & 2.7133145255 \\
\hline H80 & -3.4009841485 & -2.9132352625 & 0.4795426218 \\
\hline H81 & -1.8943810152 & -1.0654103977 & -0.1692797371 \\
\hline H82 & 0.0690568695 & -1.3310949326 & 3.6502429735 \\
\hline
\end{tabular}




$\begin{array}{llll}\text { H83 } & -1.4366875158 & -3.1758331364 & 4.2942984580 \\ \text { X84 } & 0.7626760298 & 0.1113375999 & 1.5877839687\end{array}$

TS B

energy: $\quad-2483.32082974277$ hartrees

The zero point energy (ZPE): $422.626 \mathrm{kcal} / \mathrm{mol}$

final geometry:

angstroms

\begin{tabular}{|c|c|c|c|}
\hline atom & $\mathrm{x}$ & $\mathrm{y}$ & $\mathrm{z}$ \\
\hline Ir1 & 0.0149424509 & -0.0047896187 & -0.0059957166 \\
\hline $\mathrm{H} 2$ & 0.0697622002 & 0.2451076959 & 1.6475815262 \\
\hline $\mathrm{C} 3$ & 1.5065487234 & 0.1372521397 & 1.7408163602 \\
\hline $\mathrm{H} 4$ & -0.2629866618 & -0.4074631673 & -1.6481706318 \\
\hline $\mathrm{C} 5$ & 2.2012673895 & -0.0776750768 & 0.4778882437 \\
\hline H6 & 0.6188929872 & -0.2019925505 & -1.5885506563 \\
\hline $\mathrm{H} 7$ & 0.1252546401 & -1.5315668636 & 0.2761939259 \\
\hline S8 & 0.1323985388 & 4.5823467317 & -1.5825412057 \\
\hline C9 & 0.2489539735 & 3.3516895344 & -0.3507709233 \\
\hline N10 & -0.4438267310 & 2.2630372165 & -0.6393180547 \\
\hline $\mathrm{C} 11$ & -1.1886867088 & 2.4177090502 & -1.8123444915 \\
\hline $\mathrm{C} 12$ & -2.2915091446 & 1.4513499491 & -2.2110369692 \\
\hline $\mathrm{C} 13$ & -3.1921680574 & 2.1049371574 & -3.2871530582 \\
\hline C14 & -2.3816875480 & 2.8184954573 & -4.3764167913 \\
\hline $\mathrm{C} 15$ & -1.6053976579 & 4.0058272073 & -3.7804732971 \\
\hline $\mathrm{C} 16$ & -0.9654719998 & 3.5869586285 & -2.4872208417 \\
\hline H17 & -3.8609962505 & 2.8297589985 & -2.8027089825 \\
\hline H18 & -1.6795854493 & 2.1132736080 & -4.8392086416 \\
\hline H19 & -0.8467790257 & 4.3678693712 & -4.4843132355 \\
\hline $\mathrm{H} 20$ & -1.8603055638 & 0.5434473798 & -2.6562911456 \\
\hline
\end{tabular}




\begin{tabular}{|c|c|c|c|}
\hline $\mathrm{H} 21$ & -3.8311943720 & 1.3312396276 & -3.7258027483 \\
\hline $\mathrm{H} 22$ & -3.0441257105 & 3.1747066395 & -5.1712736090 \\
\hline $\mathrm{H} 23$ & -2.2854001208 & 4.8496333372 & -3.5994915209 \\
\hline $\mathrm{C} 24$ & -3.1467348568 & 1.0515398898 & -0.9821368106 \\
\hline $\mathrm{H} 25$ & -3.3645360733 & 1.9454274264 & -0.3909152368 \\
\hline $\mathrm{H} 26$ & -4.1059770930 & 0.6421959376 & -1.3141242381 \\
\hline P27 & -2.3365857931 & -0.2245488699 & 0.1062507122 \\
\hline $\mathrm{C} 28$ & -4.0435984646 & 0.1850537909 & 4.4065547569 \\
\hline $\mathrm{C} 29$ & -3.9347240446 & 1.3184414371 & 3.5993272418 \\
\hline $\mathrm{C} 30$ & -3.4518998129 & 1.2095085354 & 2.2938087542 \\
\hline C31 & -3.0790737135 & -0.0412361144 & 1.7774341821 \\
\hline C32 & -3.1848542837 & -1.1760876827 & 2.5983642864 \\
\hline C33 & -3.6644405645 & -1.0606317292 & 3.9024377154 \\
\hline H34 & -4.4217730068 & 0.2712805910 & 5.4208004285 \\
\hline H35 & -4.2309756155 & 2.2909488636 & 3.9817169120 \\
\hline H36 & -3.3735958397 & 2.1083725863 & 1.6897329474 \\
\hline H37 & -2.8935472862 & -2.1518660897 & 2.2222253822 \\
\hline H38 & -3.7433587952 & -1.9482015262 & 4.5234420911 \\
\hline C39 & -4.0919936953 & -4.1691459351 & -1.5900801922 \\
\hline C40 & -2.7148856804 & -3.9592297619 & -1.6189925049 \\
\hline $\mathrm{C} 41$ & -2.1733381586 & -2.7852951543 & -1.0917496795 \\
\hline $\mathrm{C} 42$ & -3.0072903085 & -1.8083570639 & -0.5318559873 \\
\hline C43 & -4.3958086476 & -2.0320502374 & -0.5011833488 \\
\hline $\mathrm{C} 44$ & -4.9320682900 & -3.2040919124 & -1.0278349025 \\
\hline $\mathrm{H} 45$ & -4.5125811754 & -5.0825894963 & -2.0001761429 \\
\hline H46 & -2.0565148659 & -4.7088363761 & -2.0479701332 \\
\hline $\mathrm{H} 47$ & -1.0996117924 & -2.6364774632 & -1.1072039589 \\
\hline H48 & -5.0601536102 & -1.2978604750 & -0.0526275207 \\
\hline H49 & -6.0053103978 & -3.3672261662 & -0.9964628926 \\
\hline C50 & 1.9599674763 & 4.4716734357 & 3.3973096834 \\
\hline C51 & 0.8102646555 & 3.6791397024 & 3.3541119990 \\
\hline
\end{tabular}




\begin{tabular}{|c|c|c|c|}
\hline C52 & 0.2783808912 & 3.2841444984 & 2.1282779557 \\
\hline $\mathrm{C} 53$ & 0.9011790871 & 3.6702031901 & 0.9271116558 \\
\hline C54 & 2.0526056663 & 4.4710293955 & 0.9799122666 \\
\hline C55 & 2.5765748281 & 4.8661233883 & 2.2085998598 \\
\hline H56 & 2.3685221990 & 4.7858935682 & 4.3533960827 \\
\hline H57 & 0.3156947897 & 3.3847196528 & 4.2755273282 \\
\hline H58 & -0.6368084570 & 2.7011386872 & 2.0922227144 \\
\hline H59 & 2.5531377448 & 4.7613233885 & 0.0610969739 \\
\hline H60 & 3.4719404309 & 5.4795727862 & 2.2381538154 \\
\hline H61 & 1.6374875337 & 1.1287985089 & 2.1709581438 \\
\hline C62 & 2.8036870186 & -1.4582076628 & 0.1966163739 \\
\hline H63 & 2.9626339992 & -1.5963595389 & -0.8756385169 \\
\hline H64 & 3.7870084419 & -1.5273244508 & 0.6800426217 \\
\hline H65 & 2.1777910508 & -2.2743113768 & 0.5620313703 \\
\hline C66 & 5.0617903056 & 2.8603608831 & -0.8726948094 \\
\hline C67 & 5.0427767549 & 2.4343860072 & 0.4555362643 \\
\hline C68 & 4.0773840532 & 1.5244500606 & 0.8845598559 \\
\hline C69 & 3.1112579250 & 1.0246790407 & -0.0041646412 \\
\hline $\mathrm{C} 70$ & 3.1473835739 & 1.4541839840 & -1.3368814249 \\
\hline C71 & 4.1098421196 & 2.3675922209 & -1.7673309758 \\
\hline $\mathrm{H} 72$ & 5.8160499123 & 3.5653591018 & -1.2103927044 \\
\hline H73 & 5.7846070173 & 2.8030418182 & 1.1586295588 \\
\hline H74 & 4.0878357116 & 1.1759317974 & 1.9144879355 \\
\hline $\mathrm{H} 75$ & 2.4130189608 & 1.0761308870 & -2.0432776674 \\
\hline H76 & 4.1195332993 & 2.6914920937 & -2.8042412145 \\
\hline $\mathrm{C} 77$ & 1.5116811580 & -0.9704044989 & 2.7872679064 \\
\hline $\mathrm{O} 78$ & 2.5696293345 & -0.7723449439 & 3.5891160251 \\
\hline O79 & 0.7264710530 & -1.8835762335 & 2.8831663350 \\
\hline $\mathrm{C} 80$ & 2.7682381952 & -1.7664841931 & 4.6222827229 \\
\hline H81 & 3.6521425060 & -1.4423485647 & 5.1695615517 \\
\hline H82 & 1.8968719677 & -1.8071122219 & 5.2790378614 \\
\hline
\end{tabular}


TS C

energy: $\quad-2483.33740711748$ hartrees

The zero point energy (ZPE): $423.079 \mathrm{kcal} / \mathrm{mol}$

final geometry:

angstroms

\begin{tabular}{|c|c|c|c|}
\hline atom & X & $\mathrm{y}$ & $\mathrm{Z}$ \\
\hline $\mathrm{H} 1$ & 0.0392672746 & 0.0202060089 & -0.0538058791 \\
\hline $\mathrm{C} 2$ & 0.0182325058 & 0.0435169267 & 1.3420212352 \\
\hline Ir3 & 1.7340408307 & -0.0078175877 & -0.2960895712 \\
\hline S4 & 1.7125262157 & -4.7585716156 & 0.4497428413 \\
\hline $\mathrm{C} 5$ & 2.2993521807 & -3.1251584307 & 0.5108478759 \\
\hline N6 & 1.7368563297 & -2.3338028035 & -0.3897121335 \\
\hline $\mathrm{C} 7$ & 0.8394743404 & -3.0314668970 & -1.2052455703 \\
\hline $\mathrm{C} 8$ & 0.1479459557 & -2.4050570384 & -2.4031073257 \\
\hline C9 & -0.5253466789 & -3.5027988534 & -3.2643273026 \\
\hline $\mathrm{C} 10$ & -1.2463804504 & -4.5687412707 & -2.4325570522 \\
\hline $\mathrm{C} 11$ & -0.2426752589 & -5.3317079594 & -1.5523717460 \\
\hline $\mathrm{C} 12$ & 0.6747935805 & -4.3507963716 & -0.8766308564 \\
\hline H13 & 0.2441016495 & -3.9911896298 & -3.8782092830 \\
\hline $\mathrm{H} 14$ & -2.0068687542 & -4.0948334413 & -1.7993238633 \\
\hline H15 & -0.7625576125 & -5.9462084911 & -0.8082369163 \\
\hline H16 & -0.6380121023 & -1.7250711851 & -2.0415226646 \\
\hline H17 & -1.2207028479 & -3.0191663041 & -3.9587298374 \\
\hline H18 & -1.7712018826 & -5.2706836496 & -3.0880185524 \\
\hline H19 & 0.3456665198 & -6.0264420929 & -2.1674991678 \\
\hline $\mathrm{C} 20$ & 1.1304490319 & -1.6039645659 & -3.2929049150 \\
\hline
\end{tabular}




\begin{tabular}{|c|c|c|c|}
\hline $\mathrm{H} 21$ & 2.0826252671 & -2.1374334882 & -3.3757972933 \\
\hline $\mathrm{H} 22$ & 0.7247535789 & -1.5149639021 & -4.3054026210 \\
\hline $\mathrm{P} 23$ & 1.4637170246 & 0.0944036922 & -2.6376154040 \\
\hline $\mathrm{C} 24$ & 5.2462004951 & 1.6635747096 & -4.8174765857 \\
\hline $\mathrm{C} 25$ & 5.2386828496 & 0.3909481930 & -4.2414721369 \\
\hline $\mathrm{C} 26$ & 4.0892440076 & -0.0856143635 & -3.6143045594 \\
\hline $\mathrm{C} 27$ & 2.9263595923 & 0.7017777405 & -3.5652762783 \\
\hline $\mathrm{C} 28$ & 2.9427169222 & 1.9783611727 & -4.1449526145 \\
\hline $\mathrm{C} 29$ & 4.0992863304 & 2.4542921964 & -4.7659422461 \\
\hline H30 & 6.1440347163 & 2.0363767356 & -5.3013243112 \\
\hline H31 & 6.1286494149 & -0.2303991496 & -4.2799180519 \\
\hline H32 & 4.1069966582 & -1.0738084967 & -3.1609001944 \\
\hline H33 & 2.0573647281 & 2.6048890011 & -4.1139964859 \\
\hline H34 & 4.1002010815 & 3.4460211483 & -5.2087865741 \\
\hline $\mathrm{C} 35$ & -2.1249935568 & 2.6004968989 & -4.1738356833 \\
\hline $\mathrm{C} 36$ & -1.7521737057 & 2.6679365811 & -2.8324576802 \\
\hline $\mathrm{C} 37$ & -0.6711676750 & 1.9172035242 & -2.3681814492 \\
\hline $\mathrm{C} 38$ & 0.0481071383 & 1.0903710425 & -3.2410946647 \\
\hline C39 & -0.3275686265 & 1.0391855635 & -4.5953700569 \\
\hline $\mathrm{C} 40$ & -1.4090135275 & 1.7867764700 & -5.0555407075 \\
\hline H41 & -2.9675237388 & 3.1829160697 & -4.5349201821 \\
\hline H42 & -2.2999487842 & 3.3058795375 & -2.1449117760 \\
\hline H43 & -0.3773707305 & 1.9790529736 & -1.3262577017 \\
\hline H44 & 0.2309852837 & 0.4298432811 & -5.3006140585 \\
\hline $\mathrm{H} 45$ & -1.6904771288 & 1.7378792129 & -6.1033106128 \\
\hline H46 & 1.7186133144 & 1.5598393434 & -0.2889524272 \\
\hline H47 & 3.3472101598 & 0.0524688966 & 0.1961439714 \\
\hline H48 & 3.3683888595 & 0.1065421512 & -0.7228534268 \\
\hline C49 & 5.4597511278 & -2.2905159232 & 3.2666423538 \\
\hline $\mathrm{C} 50$ & 5.6098851602 & -1.9726524054 & 1.9148384309 \\
\hline C51 & 4.5736875053 & -2.2238713441 & 1.0180928837 \\
\hline
\end{tabular}




\begin{tabular}{|c|c|c|c|}
\hline C52 & 3.3692066631 & -2.7893088473 & 1.4688637473 \\
\hline C53 & 3.2300243791 & -3.1207817341 & 2.8269315025 \\
\hline C54 & 4.2719547883 & -2.8676193984 & 3.7188597113 \\
\hline H55 & 6.2702468266 & -2.0963032104 & 3.9629674253 \\
\hline H56 & 6.5407045730 & -1.5427188135 & 1.5566624109 \\
\hline H57 & 4.7068821811 & -2.0175310474 & -0.0394589352 \\
\hline H58 & 2.2972831897 & -3.5487083218 & 3.1818476972 \\
\hline H59 & 4.1546360231 & -3.1209454941 & 4.7684020814 \\
\hline C60 & 1.3719170846 & 0.1141787755 & 1.8869885410 \\
\hline H61 & 1.6799701590 & -0.8368914949 & 2.3099939968 \\
\hline X62 & 1.9712341286 & -0.7890866617 & 1.5748646991 \\
\hline C63 & -0.9573632146 & 1.2101251139 & 1.5059920715 \\
\hline H64 & -1.8512257809 & 1.0694614276 & 0.8977630826 \\
\hline H65 & -1.2571738292 & 1.2554343068 & 2.5591590898 \\
\hline H66 & -0.4904742615 & 2.1597875231 & 1.2545760898 \\
\hline X67 & 0.6444392031 & 0.1108834623 & 1.6383958172 \\
\hline C68 & 2.9595536896 & 3.2902502641 & 4.3419601050 \\
\hline C69 & 3.0043282144 & 1.9564019858 & 4.7448018163 \\
\hline $\mathrm{C} 70$ & 2.4905422095 & 0.9580743924 & 3.9183469652 \\
\hline C71 & 1.9159232805 & 1.2644413017 & 2.6717803598 \\
\hline $\mathrm{C} 72$ & 1.8948989495 & 2.6137127823 & 2.2754629272 \\
\hline C73 & 2.4055989728 & 3.6120843243 & 3.1016612009 \\
\hline H74 & 3.3598158084 & 4.0708923270 & 4.9822025792 \\
\hline H75 & 3.4400758603 & 1.6899672531 & 5.7036516107 \\
\hline H76 & 2.5328535722 & -0.0785978732 & 4.2425541246 \\
\hline H77 & 1.5086643295 & 2.8904587234 & 1.3007732276 \\
\hline $\mathrm{H} 78$ & 2.3784794431 & 4.6462707542 & 2.7701078587 \\
\hline C79 & -0.6459388840 & -1.3311355349 & 1.5461292727 \\
\hline O80 & -1.8347515720 & -1.3910039887 & 0.9275590718 \\
\hline O81 & -0.1785695869 & -2.2338771257 & 2.2041022051 \\
\hline $\mathrm{C} 82$ & -2.5890165420 & -2.6042284994 & 1.1455839968 \\
\hline
\end{tabular}




$\begin{array}{llll}\text { H83 } & -3.5217516995 & -2.4691365489 & 0.5994407196 \\ \text { H84 } & -2.0342548944 & -3.4655317646 & 0.7670703609 \\ \text { H85 } & -2.7807797150 & -2.7413145093 & 2.2120216614\end{array}$

\section{TS D}

energy: $\quad-2483.33038680870$ hartrees

The zero point energy (ZPE): $423.015 \mathrm{kcal} / \mathrm{mol}$

final geometry:

angstroms

$\begin{array}{lccc}\text { atom } & \mathrm{x} & \mathrm{y} & \mathrm{z} \\ \text { C1 } & -0.1380703542 & -0.1205800363 & -0.0023240738 \\ \text { H2 } & 0.0278977931 & -0.1381816065 & 1.4035174435 \\ \text { Ir3 } & 1.7170977252 & -0.0011725654 & 1.4321681284 \\ \text { H4 } & 3.2714506071 & 0.0362267807 & 0.7472745459 \\ \text { H5 } & 3.3929005966 & 0.3018652987 & 1.6038590820 \\ \text { C6 } & 1.1181008554 & 0.1318305662 & -0.7059123845 \\ \text { H7 } & 1.5901675087 & 1.5487796116 & 1.3000529663 \\ \text { S8 } & 2.3680672112 & -4.8060480713 & 1.3979519263 \\ \text { C9 } & 1.3851224100 & -3.3638728321 & 1.3887644433 \\ \text { N10 } & 2.0078916197 & -2.3155851215 & 1.9069765585 \\ \text { C11 } & 3.2482273887 & -2.6630542129 & 2.4517436416 \\ \text { C12 } & 4.0140178348 & -1.7373756888 & 3.3804573569 \\ \text { C13 } & 5.1320818390 & -2.5274511489 & 4.1060385775\end{array}$




\begin{tabular}{|c|c|c|c|}
\hline C14 & 5.8812960464 & -3.4818290538 & 3.1677217900 \\
\hline $\mathrm{C} 15$ & 4.9351777525 & -4.5781382975 & 2.6480612491 \\
\hline $\mathrm{C} 16$ & 3.6376677346 & -3.9532962411 & 2.2208038532 \\
\hline H17 & 4.6868163947 & -3.1080777157 & 4.9257781320 \\
\hline H18 & 6.2965806320 & -2.9213906435 & 2.3204589900 \\
\hline H19 & 5.3912271430 & -5.1215283572 & 1.8122289060 \\
\hline $\mathrm{H} 20$ & 4.5017522073 & -0.9386491859 & 2.8058587067 \\
\hline $\mathrm{H} 21$ & 5.8240445437 & -1.8142265262 & 4.5663140862 \\
\hline $\mathrm{H} 22$ & 6.7259563634 & -3.9413234029 & 3.6901154217 \\
\hline $\mathrm{H} 23$ & 4.7488367256 & -5.3222273710 & 3.4346505953 \\
\hline $\mathrm{C} 24$ & 3.0812170457 & -1.0957227713 & 4.4379329046 \\
\hline $\mathrm{H} 25$ & 2.5389818521 & -1.8875851743 & 4.9623635800 \\
\hline $\mathrm{H} 26$ & 3.6889240540 & -0.5801603336 & 5.1882436415 \\
\hline P27 & 1.8535259050 & 0.1610774329 & 3.7918357743 \\
\hline $\mathrm{C} 28$ & -2.1901928137 & -0.4827971482 & 5.9837618455 \\
\hline $\mathrm{C} 29$ & -1.3414964254 & -1.5710714236 & 5.7831280891 \\
\hline C30 & -0.1099423809 & -1.3949965725 & 5.1484288019 \\
\hline C31 & 0.2884384855 & -0.1221188721 & 4.7133691710 \\
\hline C32 & -0.5763086016 & 0.9671120050 & 4.9151843214 \\
\hline C33 & -1.8054341298 & 0.7859923977 & 5.5457807304 \\
\hline H34 & -3.1462924235 & -0.6209472680 & 6.4799587840 \\
\hline H35 & -1.6332661345 & -2.5608107980 & 6.1218499174 \\
\hline H36 & 0.5248467519 & -2.2617025487 & 4.9943414305 \\
\hline H37 & -0.2855008230 & 1.9622346281 & 4.5917310386 \\
\hline H38 & -2.4605939501 & 1.6383965992 & 5.7003137606 \\
\hline C39 & 3.5157089187 & 4.1313117684 & 5.5203138874 \\
\hline $\mathrm{C} 40$ & 3.4758141060 & 3.9445933937 & 4.1398137684 \\
\hline C41 & 2.9599390983 & 2.7615486531 & 3.6063327313 \\
\hline C42 & 2.4793020686 & 1.7540218157 & 4.4530222105 \\
\hline C43 & 2.5238931616 & 1.9507217814 & 5.8455343473 \\
\hline $\mathrm{C} 44$ & 3.0389640339 & 3.1318781758 & 6.3730131921 \\
\hline
\end{tabular}




\begin{tabular}{|c|c|c|c|}
\hline H45 & 3.9146121254 & 5.0528817398 & 5.9339260238 \\
\hline H46 & 3.8420909152 & 4.7194853236 & 3.4728623010 \\
\hline H47 & 2.9256083540 & 2.6267967820 & 2.5308031559 \\
\hline $\mathrm{H} 48$ & 2.1424544260 & 1.1884924443 & 6.5197465604 \\
\hline H49 & 3.0638670669 & 3.2753553037 & 7.4492600893 \\
\hline $\mathrm{C} 50$ & -2.7652598569 & -3.8156123665 & 0.4819774815 \\
\hline C51 & -2.3602901161 & -3.0233192430 & 1.5574479599 \\
\hline C52 & -1.0038615159 & -2.8546769585 & 1.8298889694 \\
\hline C53 & -0.0350863384 & -3.4716142411 & 1.0196085046 \\
\hline C54 & -0.4511404353 & -4.2741433370 & -0.0552264913 \\
\hline C55 & -1.8073637975 & -4.4410596712 & -0.3202890868 \\
\hline H56 & -3.8225706684 & -3.9488102819 & 0.2714665184 \\
\hline H57 & -3.0992071559 & -2.5435065400 & 2.1927102354 \\
\hline H58 & -0.6925724064 & -2.2610909697 & 2.6824330544 \\
\hline H59 & 0.2884424952 & -4.7301619576 & -0.7050666193 \\
\hline H60 & -2.1187753523 & -5.0533699809 & -1.1611877227 \\
\hline H61 & -0.4564963215 & -1.1580037894 & -0.0855462114 \\
\hline C62 & 1.3675455434 & 1.4867472656 & -1.3570515087 \\
\hline H63 & 2.4340406268 & 1.6805756250 & -1.4758128545 \\
\hline H64 & 0.9174370052 & 1.5052246974 & -2.3579716170 \\
\hline H65 & 0.9234678009 & 2.2953513363 & -0.7768305089 \\
\hline C66 & -3.4243759224 & 2.6476591383 & -0.3316776248 \\
\hline C67 & -3.3246165637 & 1.5316618031 & -1.1643145461 \\
\hline C68 & -2.2616387747 & 0.6413587402 & -1.0200001039 \\
\hline C69 & -1.2767546764 & 0.8666520174 & -0.0466833026 \\
\hline C70 & -1.3884706385 & 1.9799914114 & 0.7913821818 \\
\hline C71 & -2.4572988473 & 2.8659914362 & 0.6487604078 \\
\hline H72 & -4.2548603460 & 3.3386971536 & -0.4429583437 \\
\hline H73 & -4.0777653078 & 1.3503042191 & -1.9255938586 \\
\hline H74 & -2.1937143437 & -0.2299812936 & -1.6664731235 \\
\hline H75 & -0.6410402226 & 2.1482706374 & 1.5603527628 \\
\hline
\end{tabular}




$\begin{array}{llll}\text { H76 } & -2.5329827062 & 3.7274000379 & 1.3063108308 \\ \text { C77 } & 1.6729860793 & -1.0503171771 & -1.4578037544 \\ \text { O78 } & 2.7355047468 & -0.6981968993 & -2.2080082646 \\ \text { O79 } & 1.2333857412 & -2.1852273399 & -1.4235653044 \\ \text { C80 } & 3.3020890477 & -1.7479052695 & -3.0164923368 \\ \text { H81 } & 4.1172894495 & -1.2817707939 & -3.5687744209 \\ \text { H82 } & 3.6760377279 & -2.5585678352 & -2.3864743526 \\ \text { H83 } & 2.5514182029 & -2.1474503858 & -3.7022270536 \\ \text { H84 } & 0.4883633559 & 0.0052933393 & -0.3531929944\end{array}$

